\title{
Open ocean regimes of relative dispersion
}

\author{
By MICHEL OLLITRAULT ${ }^{1}$, CÉLINE GABILLET ${ }^{2}$ \\ AND ALAIN COLIN DE VERDIÈRE \\ ${ }^{1}$ Laboratoire de Physique des Océans - IFREMER Centre de Brest, BP 70, 29280 Plouzané, France \\ ${ }^{2}$ Institut de Recherche de l'Ecole Navale, Lanvéoc Poulmic BP 600, 29240 Brest Naval, France \\ ${ }^{3}$ Laboratoire de Physique des Océans, Université de Bretagne Occidentale BP 80929285 Brest, France
}

(Received 21 June 2002 and in revised form 26 January 2005)

As two fluid particles separate in time, the entire spectrum of eddy motions is being sampled from the smallest to the largest scales. In large-scale geophysical systems for which the Earth rotation is important, it has been conjectured that the relative diffusivity should vary respectively as $D^{2}$ and $D^{4 / 3}$ for distances respectively smaller and larger than a well-defined forcing scale of the order of the internal Rossby radius (with $D$ the r.m.s. separation distance). Particle paths data from a mid-latitude float experiment in the central part of the North Atlantic appear to support these statements partly: two particles initially separated by a few $\mathrm{km}$ within two distinct clusters west and east of the mid-Atlantic ridge, statistically dispersed following a Richardson regime ( $D^{2} \sim t^{3}$ asymptotically) for r.m.s. separation distances between 40 and $300 \mathrm{~km}$, in agreement with a $D^{4 / 3}$ law. At early times, and for smaller separation distances, an exponential growth, in agreement with a $D^{2}$ law, was briefly observed but only for the eastern cluster (with an e-folding time around 6 days). After a few months or separation distances greater than $300 \mathrm{~km}$, the relative dispersion slowed down naturally to the Taylor absolute dispersion regime.

\section{Introduction}

Observations of the separation of pairs of particles is one of the few experimental methods available to examine the spatial structure of geophysical turbulent flows. Richardson (1926) proposed that the relative diffusivity of an ensemble of pairs should scale as the 4/3 power of the (r.m.s.) separation distance. In his review of the subject, Corrsin (1962) emphasized the concept that turbulent eddies much smaller or much larger than the separation scale are relatively inefficient in further separation at the difference of eddies near the separation scale: the small eddies cause independent random walks of each member of the pair while the larger ones move the pair coherently as a single unit. As a result the relative velocity of the pair changes with the separation and is a non-stationary random variable. This accelerating property of relative diffusion has been used to infer properties of the energy wavenumber spectrum in the inertial range. The key quantity in the inertial range of three-dimensional turbulence is the energy dissipation rate. When the relative diffusivity is assumed to depend only on this energy dissipation rate and on separation, Richardson's law is recovered (Obukhov 1941; Batchelor 1952). The study of particle dispersion is important because of the interest in transport and mixing of chemicals in large-scale geophysical systems. While Taylor's (1921) single-particle dispersion theory relates to tracer dispersal from a fixed geographical origin and for very large times, two-particle dispersion relates to the spreading of a cloud of tracer from its centre of gravity (Batchelor 1952) and will 
give information on the cloud size growth as time increases. Relative dispersion thus describes the fate of a passive tracer much better than one-particle dispersion.

The subject has now branched into two, with the dispersive effects of threedimensional high Reynolds number turbulence on the one hand and those of twodimensional turbulence on the other. The former has been recently reviewed (Sawford 2001). Because the oceanic motions that separate the floats in our experiment are associated with a small Rossby number (horizontal characteristic length scale $L$ greater than $1 \mathrm{~km}$ ), the latter context seems more appropriate, although it is only under certain circumstances (to be detailed below) that the flow can be considered approximately two-dimensional. In two-dimensional free turbulence, the square of relative vorticity is conserved following fluid particles and enstrophy (or mean square relative vorticity) cascades to small scales while energy moves up scale as a result of nonlinear interactions (Batchelor 1953). Similarly, Kraichnan (1967) showed that two-dimensional forced turbulence admits two formal inertial ranges, one on each side of a given injection scale, with an inverse energy cascade at scales larger than the injection scale and a direct enstrophy cascade at smaller scales. He then proposed that the same arguments used in the three-dimensional case to infer the $4 / 3$ law could be applied to the region of the inverse energy cascade, an idea which has been supported by various turbulence closure calculations (Kraichnan 1966; Larcheveque \& Lesieur 1981).

In the inertial range of the enstrophy cascade, Lin (1972) proposed that relative dispersion should depend only on the separation and on the enstrophy dissipation rate with the consequence that at such scales the relative diffusivity varies as the square of the separation. This in turn implies that the mean-square separation grows exponentially in time. Kraichnan \& Montgomery (1980) note in their review of twodimensional turbulence that separation is then smaller than the size of the eddies that carry most of the shear. This approximates the situation of spatially uniform random shear with the rate of separation proportional to the separation. Larcheveque \& Lesieur (1981) confirmed the validity of this regime under an eddy damped quasinormal Markovian (EDQNM) closure. Numerical experiments in the framework of two-dimensional homogeneous decaying turbulence carried out by Kowalsky \& Peskin (1981), did show a short exponential regime (with a ratio of the initial r.m.s. separation to the injection scale of 0.035). However, Babiano et al. (1990) who conducted similar numerical experiments, following Kraichnan's assumptions of forcing at a well-defined wavenumber, observed that the exponential law was "reduced to a very short transient stage". On the other hand they found that the $t^{3}$ asymptotic law, a consequence of Richardson's law, was obtained "provided the initial separation of the particles is smaller by an order of magnitude than the injection scale", a condition which looks surprising and will be discussed below. An experimental study of the dispersion of pairs of passive particles in a stratification controlled two-dimensional laboratory flow was performed recently by Jullien, Paret \& Tabeling (1999), the local forcing being provided by the interaction of an electrical current with a magnetic field. Within the inertial range of the inverse cascade (i.e. at scales larger than the forcing scale), the $t^{3}$ law was observed along with a strongly non-Gaussian behaviour for the probability distribution of pair separations (with initial separations much smaller than the injection scale).

The application of these two-dimensional physical concepts to large-scale geophysical rotating and stratified flows (e.g. in the ocean or atmosphere) has no definite results yet (see the reviews by Rhines 1979, and Salmon 1998). If the flow time scale is much greater than $f^{-1}$, and the flow horizontal length scale $L$ much smaller than $f \beta^{-1}(f=2 \Omega \sin \varphi$ and $\beta=\mathrm{d} f / a \mathrm{~d} \varphi$, with $\Omega$ the Earth rotation rate, $\varphi$ the latitude 
and $a$ the Earth radius), then with an horizontal velocity scale $U$ much smaller than $f L$ (Rossby number $\ll 1$ ), quasi-geostrophic potential vorticity is conserved along a quasi-horizontal trajectory (e.g. Gill 1982). If furthermore $L$ is much smaller than the internal Rossby radius of deformation $R_{\text {int }}$ (at which vortex stretching appears) and the Rhines scale $(U / \beta)^{1 / 2}$ (at which planetary waves start to propagate), the flow is effectively quasi-two-dimensional. Since $R_{\text {int }} \sim 30 \mathrm{~km}$ while $(U / \beta)^{1 / 2} \sim 70 \mathrm{~km}$ at mid-latitudes in the ocean, such a quasi-two-dimensional regime is possible and could be observed near scales of the order of a few $\mathrm{km}\left(U \sim 0.01 \mathrm{~m} \mathrm{~s}^{-1}\right.$ to $\left.0.1 \mathrm{~m} \mathrm{~s}^{-1}\right)$. In the ocean and atmosphere the energy source for the meso- (or synoptic) scale eddies comes mainly, through baroclinic instability, from the potential energy associated with the sloping isopycnals of the semi-permanent jets (Gill 1982). Eddy kinetic energy is injected preferentially at the scale (scale being defined as wavelength $/ 2 \pi$ ) of the internal Rossby radius of deformation $R_{\text {int }}$ which would thus play the role of Kraichnan's injection scale. However, in the atmosphere, the range of horizontal scales over which the quasi-two-dimensional regime could exist may be narrower than in the ocean, since atmospheric values for $R_{\text {int }}$ and $(U / \beta)^{1 / 2}$ are both of the order of $700 \mathrm{~km}$ while the condition of a small Rossby number imposes scales greater than $100 \mathrm{~km}$, for speed of $\sim 1 \mathrm{~m} \mathrm{~s}^{-1}$.

Discarding the $\beta$ effect (thus for horizontal scales much smaller than the Rhines scale), but keeping the vertical stretching term in the quasi-geotrophic potential vorticity, Charney (1971) has shown that for scales smaller (not necessarily much smaller) than $R_{\text {int }}$, a potential enstrophy inertial range can exist under certain conditions, which renders Lin's law for pair dispersion still plausible with potential enstrophy dissipation rate as the leading controlling parameter.

At scales larger than $R_{\text {int }}$, and for uniform stratification, Rhines (1979) has argued that an inverse energy cascade is still possible in the horizontal and vertical directions, the cascade proceeding to a state of alternating, weakly interacting barotropic (i.e. $z$-independent) zonal jets of width $O(U / \beta)^{1 / 2}$. There is no observational evidence that the oceanic eddies equilibrate similarly. Stammer (1997) has shown that eddies at the surface scale with $R_{i n t}$ and not with $(U / \beta)^{1 / 2}$ while equipartition of the first baroclinic mode and barotropic mode energy is usually observed at mid-latitudes (Wunsch 1997). On the modelling side small-scale topography (Tréguier \& Hua 1988) or realistic oceanic stratification (Smith \& Vallis 2001, 2002) have been shown to be serious candidates to prevent the inverse cascade reaching a full barotropic state. The applicability of the Richardson-Kraichnan law to realistic regimes of oceanic turbulence (beyond the two-dimesional hypothesis) thus remains open giving added value to any experimental evidence that might be gained on such an issue.

The EOLE experiment was the first geophysical experiment aimed at testing these laws of two-dimensional relative dispersion in the mid-latitude atmosphere and Morel \& Larcheveque (1974) observed the initial exponential time regime of the enstrophy cascade. In the TWERL experiment, Er El \& Peskin (1981) confirmed this exponential regime but the demonstration of a Richardson regime at larger scale proved elusive. These authors emphasized the importance of looking at the dispersion in the time domain, a proposal that we share: we will show the differences that arise when averages of pair diffusivity are constructed as a function of distance or time. Assuming that the strain rate separating a pair was dominated by eddy scales near the separation distance, Morel \& Larcheveque (1974) concluded that the $D^{2}$ law for the relative diffusivity was evidence of the existence of a $k^{-3}$ energy spectrum in the enstrophy inertial range. This conclusion was disputed by Bennett (1984) and Babiano, Basdevant \& Sadourny (1985) who argued that such a diffusivity law implies only 
that the slope of the energy spectrum is steeper than or equal to -3 . When the slope is steeper than -3 , the separation process is said to be non-local meaning that eddies of scales larger than the separation also contribute to the diffusivity. Bennett (1984) describes the two effects: local straining causing a blob of contaminant to become highly convoluted with significant cross-diffusion before the blob envelope reaches the scale of the energy-containing eddies and non-local straining causing the blob to be drawn into a few long streaks with little cross-diffusion.

Summarizing the oceanic experimental context some thirty years ago, Okubo (1971) found that the $D^{4 / 3}$ law for the relative diffusivity along with the $t^{3}$ law for the meansquare separation held for a range of scales from $10 \mathrm{~m}$ to $300 \mathrm{~km}$, the fit being local in the sense that the proportionality constant, the energy dissipation rate, had to be varied to accommodate this large spectral breadth. Most of the data in Okubo's diffusion diagrams came from surface dye releases in coastal areas where processes as varied as wind waves, tidal and inertial fluctuations are present. As Okubo points out, the similarity theory of turbulence is one possibility among others to rationalize the data (but he did not say whether he meant the two- or three-dimensional version of that theory). He \& Bennett (1987) drew attention to the combination of mean shear and turbulent mixing as another possible cause for the $t^{3}$ law.

Although float observations have become common practice to infer the largescale oceanic circulation, investigations of turbulent dispersion revealed by the floats have concerned for the most part one-particle (or so-called absolute) dispersion. McWilliams et al. (1983) showed an example (calculated by Price) from the Local Dynamics Experiment (LDE), with floats at depth of $700 \mathrm{~m}$ approximately, in the North West Atlantic $\left(\sim 31^{\circ} \mathrm{N}, \sim 70^{\circ} \mathrm{W}\right)$, supporting a $D^{4 / 3}$ law for separation distances between 70 and $300 \mathrm{~km}$, and suggesting a $D^{2}$ law for smaller separations. Recently Lacasce \& Bower (2000) computed relative dispersion from four mid-latitude float experiments (including LDE) in the North Atlantic for three classes of initial separations $\left(D_{0} \leqslant 7.5 \mathrm{~km}, \leqslant 15 \mathrm{~km}\right.$ and $\leqslant 30 \mathrm{~km}$ respectively): relative dispersion in the two Western sites was shown to follow roughly a 4/3 law for r.m.s. separations in the $10-100 \mathrm{~km}$ range, but exponential stretching was not resolved. For the two other regions (ACCE and AMUSE experiments), relative dispersion varied linearly with separation instead, a result interpreted by the authors to be the signature of a stochastic mixing process with particles essentially uncorrelated in space. Finally, using 25 day trajectories from surface drifters deployed in the Gulf of Mexico, Lacasce \& Ohlmann (2003) obtained, for small initial separations $\left(D_{0} \leqslant 1 \mathrm{~km}\right)$ an exponential growth over the time range 2 to 10 days after launch, corresponding to separation distances between 5 and $40 \mathrm{~km}$.

The TOPOGULF experiment whose data is used in the present paper was carried out in the central interior of the subtropical gyre of the North Atlantic away from boundary currents. Acoustically tracked subsurface floats were deployed at $700 \mathrm{~m}$ depth in the main thermocline (Ollitrault \& Colin de Verdière 2002a) at scales sufficiently small initially to allow tests of the various laws of relative dispersion against distance or time chosen as independent variables respectively. More specifically this data set should allow the following questions to be answered:

Is there evidence of an exponential regime of relative dispersion?

Is there evidence of a transition to a Richardson regime before the members of a given pair randomly walk independently?

Section 2 describes briefly the float experiment. The theoretical elements needed are presented in $\S 3$. Section 4 and $\S 5$ describe respectively the statistical treatment of the data and the results. These results are further interpreted in $\S 6$. 


\section{The float experiment}

As part of the TOPOGULF experiment (Arhan, Colin de Verdière \& Mercier 1989; Colin de Verdière, Mercier \& Arhan 1989), 26 quasi-Lagrangian subsurface (SOFAR) floats were used to track water particles near $700 \mathrm{dbar}$ in the mid-latitude North Atlantic, over a 6 year (mid 1983 to mid 1989) period. Fifty three float years were collected, allowing absolute velocities and displacements for intermediate water particles from submesoscales (i.e. with characteristic length and time scales of order $10 \mathrm{~km}$ and 5 days) to mesoscales (100 km and 50 days) up to the largest scales of the circulation (in the present experiment roughly $1000 \mathrm{~km}$ and 500 days) to be obtained. Fourteen floats were launched (4-7 July 1983) within a $75 \mathrm{~km}$ diameter circle centred $36^{\circ} \mathrm{N} 40^{\circ} \mathrm{W}$, west of the Mid-Atlantic Ridge (MAR), with a near neighbour distance of $18 \mathrm{~km}$. Ten floats were launched one year later (28-29 October 1984) within a $45 \mathrm{~km}$ diameter circle centred $33^{\circ} \mathrm{N} 33^{\circ} \mathrm{W}$, east of the MAR, as two duos and two trios (two or three floats separated by $2 \mathrm{~km}$ ), with $18 \mathrm{~km}$ distance between groups. However only one trio and one duo (plus two single floats) will be used in this study since three floats equilibrated deeper than 1100 dbar after launch. Two of these three rose back into the $[600,800]$ dbar interval but much too late to be useful here. Three more floats (one duo and a single float) were released on 11 September 1985 in the same region.

Float trajectories have revealed a very turbulent circulation of the subtropical Atlantic $\left(30^{\circ} \mathrm{N}\right.$ to $\left.45^{\circ} \mathrm{N}\right)$ and an influence of the mid-Atlantic ridge (MAR) on the large-scale motions. Trajectories, mean circulation and one-particle dispersion can be found in Ollitrault \& Colin de Verdière $(2002 a, b)$ referred to as OCV from here on.

In the present context of estimating the rate of dispersion of float pairs, it is important to discuss position errors of the floats. Our SOFAR floats were positioned every $12 \mathrm{~h}$, with an accuracy of a few $\mathrm{km}$. Errors on positions are due to several causes, the most important ones being:

(i) Imperfect knowledge of the speed of sound used to convert times into distances, which are used through least-square minimization to estimate the float position.

(ii) Error in estimating the times of propagation because of the poor knowledge of the float and listening station clock drifts.

(iii) 'Bad' geometry for the least-square fit (e.g. for a float positioned well away from a cluster of listening stations, so that constant times of propagation loci intersect each other at very small angles, or even never intersect).

(iv) Imperfect separation by the listening station correlator of the different sound rays received (from a float).

For the purpose of our study however, which is to calculate the time rate of change of separation and relative velocity vectors between two given floats for separation distances less than a few hundred $\mathrm{km}$, systematic errors should cancel out approximately. To reduce any random (as opposed to systematic) errors, a low-passed filter was applied to the raw position time series to cut off periods less than $\sim 3$ days and velocities were estimated with a cubic spline fitted to the filtered positions (Ollitrault 1994). This procedure is thought to provide an accuracy of a few hundred metres for the separation vector when floats are less than a few tens of $\mathrm{km}$ apart, degrading to probably $1 \mathrm{~km}$ or more as separation increases to a few hundreds of $\mathrm{km}$. Instantaneous velocities are assumed accurate to within $1 \mathrm{~cm} \mathrm{~s}^{-1}$.

\section{Theoretical considerations}

Following Richardson's (1926) result for three-dimensional turbulence, Kraichnan $(1966,1967)$ proposed the following law for the relative diffusivity of pairs as function 
of the r.m.s. separation distance in the inverse cascade regime of two-dimensional turbulence:

$$
\frac{1}{2} \frac{\mathrm{d} D^{2}}{\mathrm{~d} t} \sim \varepsilon^{1 / 3} D^{4 / 3} \text { for } D \gg D_{I}
$$

where $D_{I}$ is a forcing injection scale and $\varepsilon$ is the energy flux to larger scales. A similar argument led Lin (1972) to propose for the inertial range associated with the enstrophy cascade of two-dimensional turbulence:

$$
\frac{1}{2} \frac{\mathrm{d} D^{2}}{\mathrm{~d} t} \sim \chi^{1 / 3} D^{2} \text { for } D \ll D_{I}
$$

where $\chi$ is the enstrophy dissipation rate (see Kraichnan \& Montgomery 1980 and Lesieur 1997 for a review of the underlying concepts). Both laws are proposed up to a dimensionless constant which can only be estimated from dispersion measurements supplemented by direct estimation of the dissipation rates themselves (Larcheveque 1983). It is readily seen that, after integration, Kraichnan-Richardson's law gives

$$
D^{2}=\left(D_{0}^{2 / 3}+\frac{2}{3} c \varepsilon^{1 / 3} t\right)^{3}
$$

while Lin's law implies exponential growth:

$$
D^{2}=D_{0}^{2} \exp \left(2 d \chi^{1 / 3} t\right)
$$

where $c$ and $d$ are the aforementionned non-dimensional constants and $D_{0}$ is the initial separation distance.

Following Babiano et al.'s (1990) presentation, the single-particle Taylor's (1921) formulation applied to the relative dispersion proceeds as follows:

$$
D^{2}\left(t, D_{0}\right)=\left\langle D^{2}\left(t, \boldsymbol{D}_{0}\right)\right\rangle=\left\langle\boldsymbol{D}\left(t, \boldsymbol{D}_{0}\right) \cdot \boldsymbol{D}\left(t, \boldsymbol{D}_{0}\right)\right\rangle
$$

is the mean-square separation distance at time $t$. Here $\boldsymbol{D}$ is the separation vector of a pair of particles (named a and $\mathrm{b}$ ), and $\langle\cdot\rangle$ denotes the ensemble average at time $t$ over all particle pairs initially separated by a given distance $D_{0}=\left\|\boldsymbol{D}_{0}\right\|$. If $\delta \boldsymbol{V}=(\mathrm{d} / \mathrm{d} t) \boldsymbol{D}=$ $\boldsymbol{V}_{a}-\boldsymbol{V}_{b}$ is the Lagrangian relative velocity vector for the same pair of particles a and $b$, then

$$
\boldsymbol{D}\left(t, \boldsymbol{D}_{0}\right)=\boldsymbol{D}_{0}+\int_{0}^{t} \delta \boldsymbol{V}\left(\tau, \boldsymbol{D}_{0}\right) \mathrm{d} \tau .
$$

The relative diffusivity can now be rewritten as

$$
\begin{aligned}
Y\left(t, D_{0}\right) & =\frac{1}{2} \frac{\mathrm{d}}{\mathrm{d} t}\left\langle D^{2}\left(t, \boldsymbol{D}_{0}\right)\right\rangle=\left\langle\boldsymbol{D}\left(t, \boldsymbol{D}_{0}\right) \cdot \delta \boldsymbol{V}\left(t, \boldsymbol{D}_{0}\right)\right\rangle \\
& =\left\langle\boldsymbol{D}_{0} \cdot \delta \boldsymbol{V}\left(t, \boldsymbol{D}_{0}\right)\right\rangle+\int_{0}^{t}\left\langle\delta \boldsymbol{V}\left(t, \boldsymbol{D}_{0}\right) \cdot \delta \boldsymbol{V}\left(\tau, \boldsymbol{D}_{0}\right)\right\rangle \mathrm{d} \tau .
\end{aligned}
$$

Relative velocities are not stationary random variables because the covariances in (2) keep their dependence on pair separation $D_{0}$ and on time after release. Using the definition of $\delta \boldsymbol{V}$ in terms of absolute velocities $\boldsymbol{V}$, the last term in (2) can be rewritten as

$$
2 K(t)-2 \int_{0}^{t}\left\langle\boldsymbol{V}_{a}(t) \cdot \boldsymbol{V}_{b}(\tau)\right\rangle \mathrm{d} \tau .
$$

$K(t)$ is the single-particle absolute diffusivity (actually $K$ is the trace of the diffusivity tensor i.e. the sum of individual diffusivities in two orthogonal directions). For large time or large initial separation compared to the energy-containing scale, the two 
members of the pair wander independently and the last term above vanishes in which case the relative diffusivity becomes just twice the single-particle absolute diffusivity provided that the covariance $\left\langle\boldsymbol{D}_{0} \cdot \delta \boldsymbol{V}\left(t, \boldsymbol{D}_{0}\right)\right\rangle$ also vanishes. This is clearly the case at the initial time, after ensemble averaging over a very large number of particle pairs placed randomly in homogeneous turbulence (Davis 1985) but also (less obviously) for later times as verified numerically by Babiano et al. (1990). However, this is unlikely in geophysical experiments (because too small a number of pairs is available). In the absence of this term, Babiano et al. (1990) have shown on pure kinematical grounds that when $t$ goes to zero (that is when the relative velocities are perfectly correlated), the relative diffusivity and the relative dispersion grow in time respectively as $t$ and $D_{0}^{2}+\left\langle\left\|\delta \boldsymbol{V}_{0}\right\|^{2}\right\rangle t^{2}=D_{0}^{2}\left(1+Z t^{2}\right.$ ), where $Z$ is the enstrophy given by $\frac{1}{2}\left\langle\| \text { curl } \boldsymbol{V} \|^{2}\right\rangle_{x}$ (spatial averaging carried out over the entire domain is denoted by $\langle\cdot\rangle_{x}$ ).

The mean-square relative velocity is also a quantity that can be estimated from observations. From its definition we have

$$
\left\langle\frac{\mathrm{d} \boldsymbol{D}}{\mathrm{d} t} \cdot \frac{\mathrm{d} \boldsymbol{D}}{\mathrm{d} t}\right\rangle=\left\langle\left\|\delta \boldsymbol{V}\left(t, \boldsymbol{D}_{0}\right)\right\|^{2}\right\rangle=\left\langle\left\|\boldsymbol{V}_{a}(t)\right\|^{2}\right\rangle+\left\langle\left\|\boldsymbol{V}_{b}(t)\right\|^{2}\right\rangle-2\left\langle\boldsymbol{V}_{a}(t) \cdot \boldsymbol{V}_{b}(t)\right\rangle .
$$

After a sufficiently long time the absolute velocities of the two particles decorrelate and the mean-square relative velocity becomes just four times the eddy kinetic energy.

\section{Data treatment}

Due to the SOFAR system of acoustic transmission used in the TOPOGULF experiment, two different floats were never located simultaneously. However, all floats were positioned within a 6 hour interval. Furthermore, in this study we consider only daily positions, because the filtering done on the raw position estimates (see §2) may invalidate any result for times smaller than a few days. As in OCV, floats were partitioned between western and eastern basins, depending whether they were launched west or east of the MAR. No float pair constructed of a western float and an eastern float happened to satisfy the different criteria used to select the pair-separationdistance time series (see below) used in this statistical study. The original 14 western (respectively 12 eastern) float position time series were combined to yield $\left(\begin{array}{c}14 \\ 2\end{array}\right)$ (respectively $\left(\begin{array}{c}12 \\ 2\end{array}\right)$ ) pair-separation-distance time series 1000 days long. Pair separation distances of these 91 (respectively 54, because 12 pairs contain no data) time series were estimated with a $10^{-5}$ relative accuracy.

Since two floats move independently from each other if the separation distance is large enough compared to the more energetic scales, there is no need to consider large $D_{0}$. In our float launching region, where $R_{\text {int }}$ is $\sim 25 \mathrm{~km}$, structures with wavelengths of the order of $150 \mathrm{~km}$ will emerge because of the instability of the Azores current. Pair separation distance versus time plots (see figures 1,2,3 and 4 and the next section) show that once two floats have separated more than $150 \mathrm{~km}$, they never come back within $30 \mathrm{~km}$ of each other (except for pairs 03-04 and 87-88 whose separations once shrank to 12 and $20 \mathrm{~km}$ respectively). In consequence, statistics will be presented only for $D_{0}$ values less than $120 \mathrm{~km}$.

At the initial time (9 July 1983 in the west, 29 October 1984 or 11 September 1985 in the east respectively), there were 32 (respectively 12) pairs with $D_{0}<30 \mathrm{~km}$, but only 4 (respectively 5) pairs with $D_{0}<5 \mathrm{~km}$. To increase the number of pairs with small $D_{0}$, 'chance' pairs could be considered as well. A 'chance' pair is created at the time (if any) the separation shrinks to a minimum of less than $50 \mathrm{~km}$, either after launch or after the float separation has exceeded $150 \mathrm{~km}$ (so that the two floats 
Western floats (91 pairs)

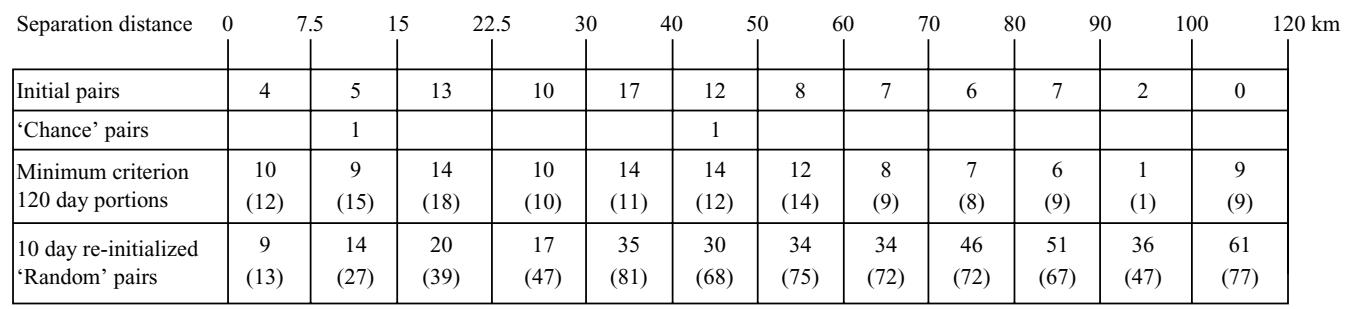

Eastern floats (54 pairs)

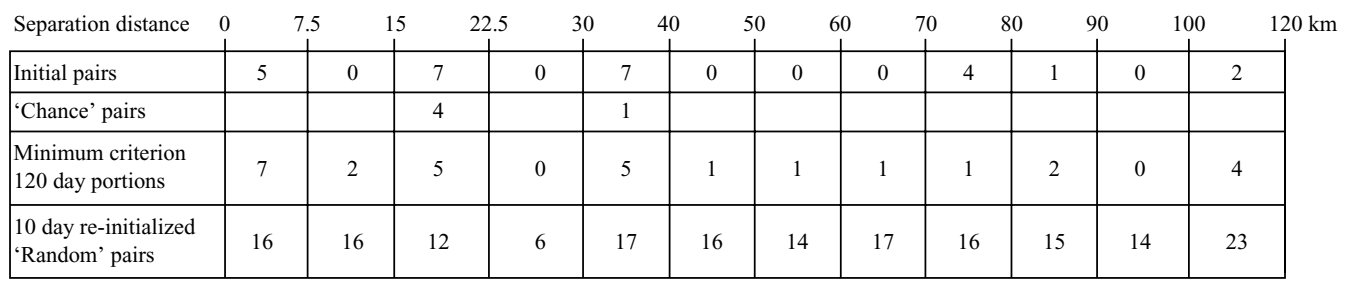

TABLE 1. Number of pairs versus separation classes. The first line contains pair numbers at the initial time: 9 July 1983 in the west; 29 October 1984 or 11 September 1985 in the east. The second line contains the 'chance' pair numbers, a 'chance' pair being defined if the separation shrinks to a minimum less than $50 \mathrm{~km}$, either after launch or after the separation overshoots $150 \mathrm{~km}$. The third line contains the pair numbers, for the minimum separation criterion and with a 120 days duration. The fourth line contains the 'random' pair numbers, a new 'random' pair being defined every 10 days from a given separation time series. For the western cluster, we have excluded roughly $50 \%$ of the data because all the floats were swirling after launch within the cyclonic eddy shown on figure 1 (the numbers of pairs, had we kept the whole data set, are given within parentheses).

can be considered quasi-independent). Table 1 shows that only seven 'chance' pairs would result with $D_{0}<50 \mathrm{~km}$. This is clearly due to the fact that floats were launched initially in clusters (see OCV) and the minimum separations occurred either at launch or soon afterwards.

Consequently we tried another approach. We searched for the minimum separation between two floats during the entire life of a pair and recorded the evolution after that minimum, over 120 days (4 months). The same procedure was used in turn on the remaining time series (search for a second minimum and selection of the subsequent 120 days). Although it approximately doubles the number of pairs for small $(<20 \mathrm{~km}) D_{0}$ (see table 1$)$, a definite advantage for the statistics, this approach selects the separation time series corresponding to hyperbolic regions. The statistical results for the time evolution of mean-square separation between two particles with the same initial separation $D_{0}$ (to be presented in the next section) would thus be biased upwards.

To preserve as far as possible a 'random' sampling of the separation process, we have finally defined the separation-distance time series as 6 month portions of a given original time series, the different portions being initialized every 10 days. This procedure clearly weakens any assumption about the statistical independence of the different portions. However, results obtained from 30 day or 60 day re-initializations (presented in the next section) substantiate this approach. The 6 month duration was selected so that the large-time random walk can be clearly reached. 
54W 53W 52W 51W 50W 49W 48W 47W 46W 45W 44W 43W 42W 41W 40W $39 \mathrm{~W} 38 \mathrm{~W}$

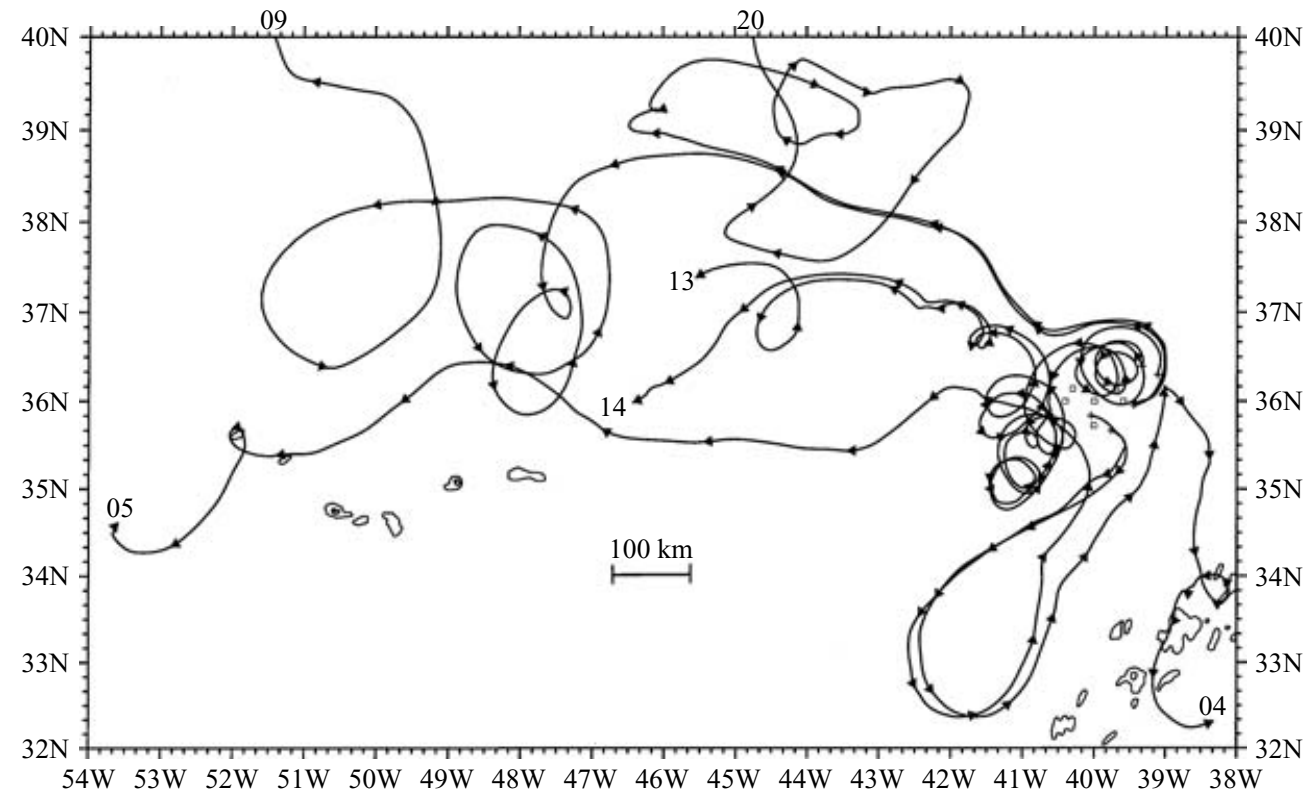

FIGURE 1. Float trajectories of three western pairs (TOPOGULF SOFAR floats 04 and 05, 13 and 14, 09 and 20) over 200 days after 9 July 1983. One arrowhead every 10 days. Floats are seen to separate swiftly once separation exceeds a few tens of $\mathrm{km}$. These floats were launched between 4 July and 7 July 1983 at positions given by squares, but their trajectories are considered only after 9 July 1983 in this study.

Basically, all the statistical estimates will be given as a function of time, because $\langle\cdot\rangle$ is a Lagrangian average. Some statistical estimates will also be given as a function of $D$ (the r.m.s. separation) since we know $D^{2}$ as a function of $t$ and $D_{0}$. But we must warn the reader that this is different from an ensemble average carried out among pairs of the same separation $D$, irrespective of time and initial separation $D_{0}$. This will be illustrated in the following section.

\section{Results}

Although individual trajectories have been presented in OCV, it may help to visualize a few pair evolutions before going into the statistics proper. Figure 1 presents three western pairs over a 200 day period. Pair 09-20 (minimum separation $1.9 \mathrm{~km}$ ) separated swiftly after 45 days, while pair 13-14 (initial separation $22 \mathrm{~km}$ ) swirled within a cold-core cyclonic eddy for 180 days before separating swiftly thereafter (the minimum distance of $7.5 \mathrm{~km}$ occurred only a few days before the swift separation, see also figure 2). Pair 04-05 (minimum separation $2.1 \mathrm{~km}$ occurred more normally a few days after launching) separated more or less similarly. Pressure differences for paired floats were less than $30 \mathrm{dbar}$, for short separations, thus minimizing any vertical shear effect on pair dispersion (figure 2). Figure 3 presents one eastern trio. As described in OCV, there is clearly a topographic influence on the flow and the float dispersion. Figure 4 gives the temperature, pressure and separation evolutions for pair $87-88$, with features similar to those of the western pairs. Since a trio gives three pairs in our analysis, it must be remarked that the three pair separations are not 

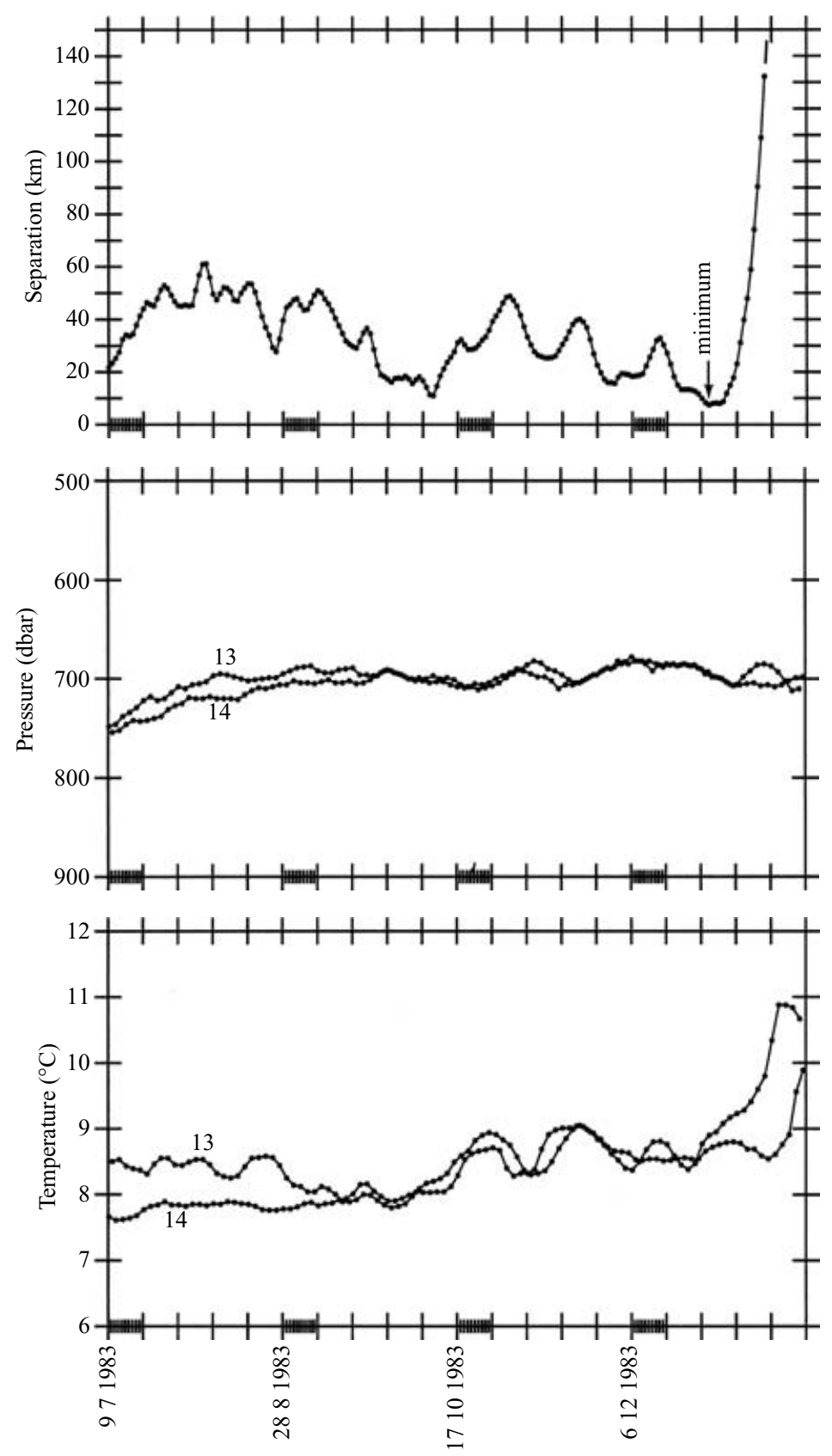

FIGURE 2. Temperature, pressure and separation time series (over 200 days after 9 July 1983) for the TOPOGULF SOFAR float pair 13-14. This pair was embedded in a cyclonic cold-core ring (where most of the 14 western floats were launched), and its separation distance oscillates between 10 and $60 \mathrm{~km}$ over 6 months after launch, as floats swirl within the ring. Absolute minimum $\left(D_{0}=7.5 \mathrm{~km}\right)$ occurs only a few days before final fast separation.

totally independent, thus giving a number of degrees of freedom for our statistical estimates smaller than the number of pairs entering the statistics. A 'chance' pair can be identified on figure 4 for the pair $87-88$. 


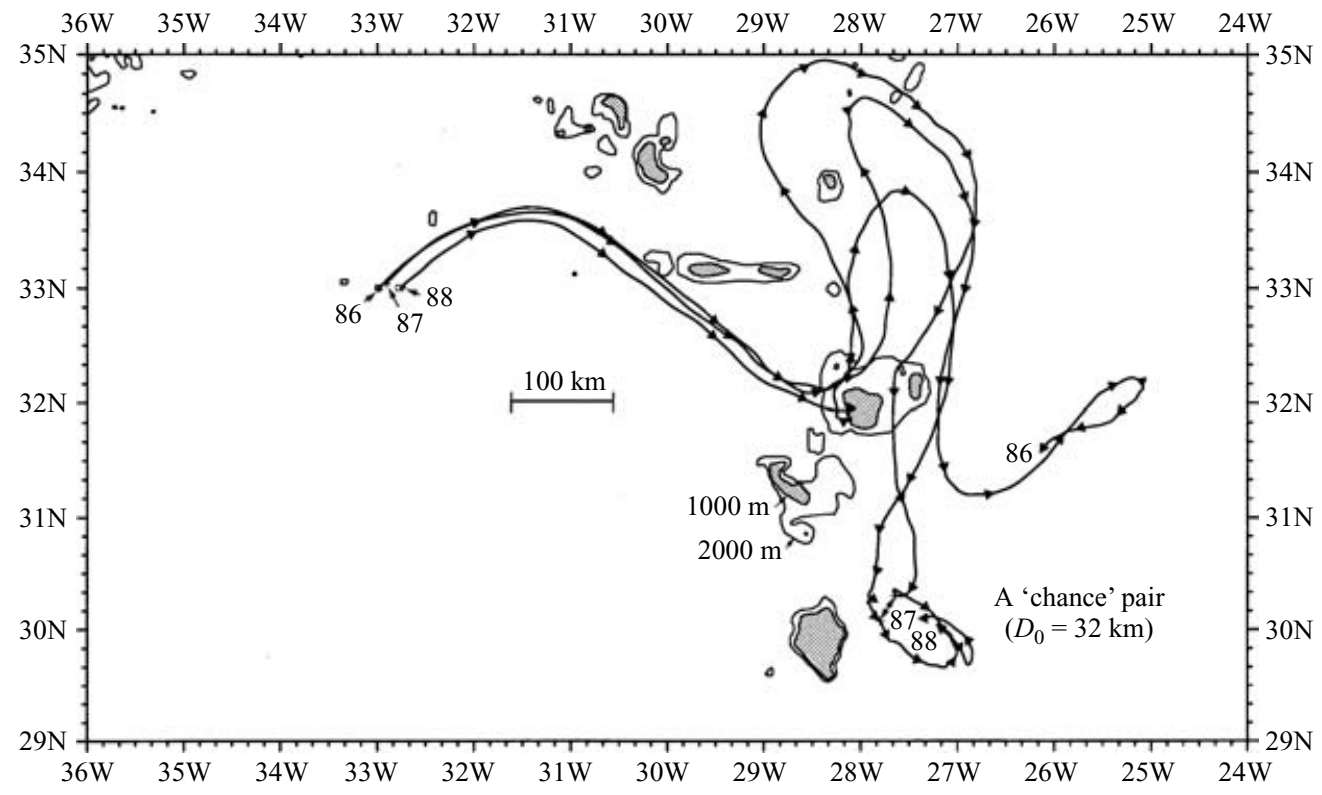

FiguRE 3. Float trajectories of a trio (TOPOGULF SOFAR floats 86, 87 and 88) over 200 days after 11 September 1985 . One arrowhead every 10 days. This float trio remained closely packed while being advected by an eastward meandering jet but dispersed as the flow impinged on the seamounts found near $28-30^{\circ} \mathrm{W}$.

We first investigated a possible anisotropy of the relative dispersion process. As shown in OCV, anisotropies appear in the single-particle diffusivities for time scales greater than a few months. Estimating the ratio of the r.m.s. zonal over r.m.s. meridional relative displacements for each class of pair separation shows a ratio close to one for scales less than a few hundreds $\mathrm{km}$ (figure 5). At greater distance, there is a tendency for zonal relative dispersion dominance, which becomes the rule near $1000 \mathrm{~km}$, in agreement with the single-particle analysis.

Restricting our interest to scales less than a few hundreds km, the mesoscale band, the results presented next are averages over all directions. Shown in figures $6(a)$ and $6(b)$ is the dispersion $D^{2}\left(t, D_{0}\right)$ as a function of time and conditional upon initial distance $D_{0}\left(D_{0}<50 \mathrm{~km}\right)$, for the western and eastern floats respectively. The classes of distance (see table 1) were chosen as a compromise between having a sufficient number of realizations within each (order of 10 or more) and being sufficiently narrow not to bias the results.

As mentioned in the previous section, the statistics obtained by initializing 'independent' portions of the pair-separation-distance time series every 10 days do not depart significantly from those obtained by initializing every 30 days or every 60 days (but they are noisier), or even from those obtained from the independent portions selected with the criterion of minimum separation (although there is a more rapid growth). A few of these results will be shown for illustration, but we mainly show the results of the 10 day re-initializations. We must also point out here that since the western floats were all launched within a cyclonic cold-core eddy, where several remained trapped for a long time (see figure 1), the results obtained with those floats should be biased towards low values. By discarding most of the floats swirling in the initial eddy, the data set for the western floats is reduced by roughly $50 \%$ compared 

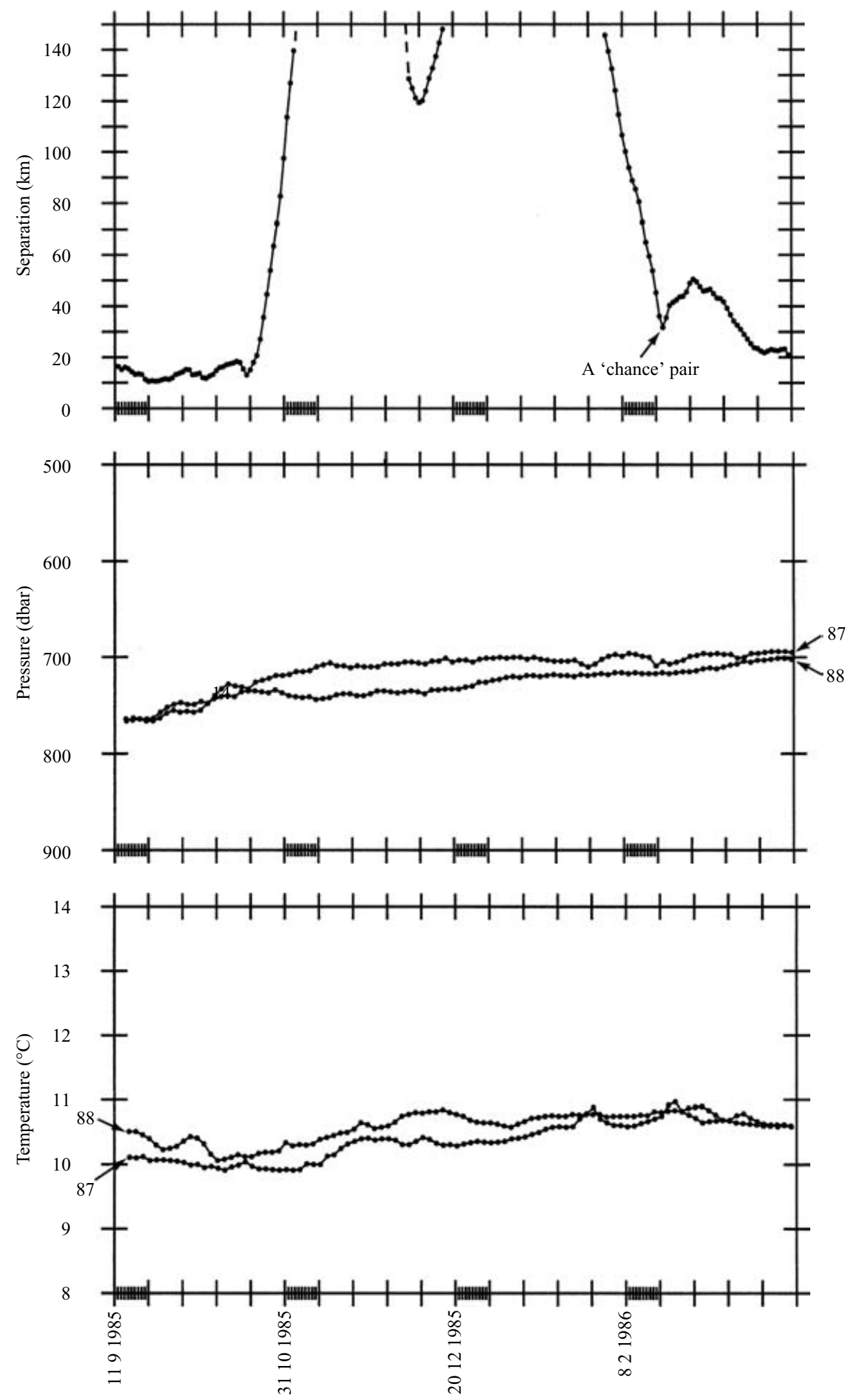

FIGURE 4. Temperature, pressure and separation time series for the TOPOGULF SOFAR float pair $87-88$. A 'chance' pair is identified near $30^{\circ} \mathrm{N} 28^{\circ} \mathrm{W}$ on February $20,1986\left(D_{0}=32 \mathrm{~km}\right)$. 

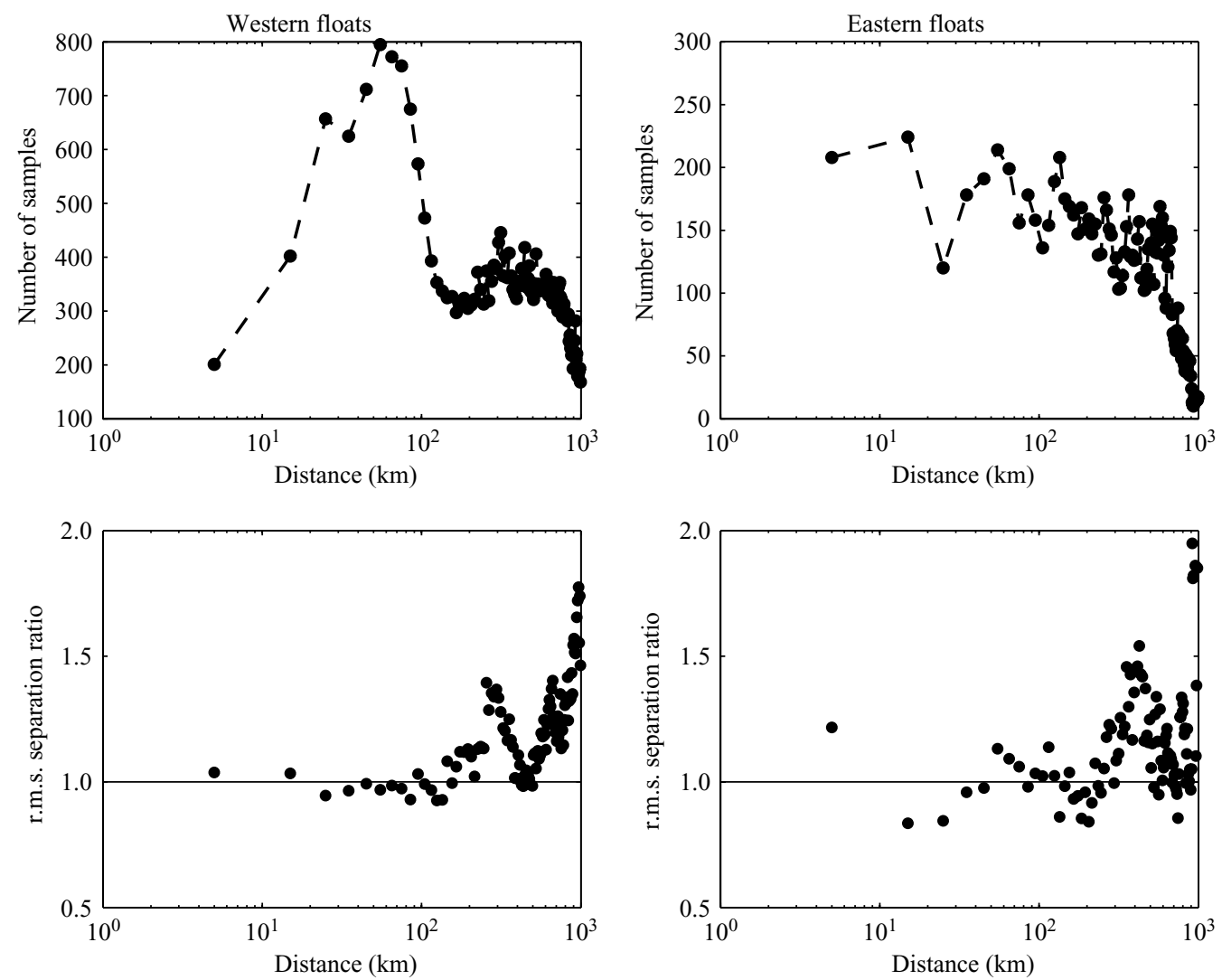

FIGURE 5. Ratio of the r.m.s. zonal and meridional separations within $10 \mathrm{~km}$ wide distance classes.

to the original set (see table 1). It is this reduced set that is used throughout this study, although some results are also given for the complete western data set.

When relative dispersion is plotted with semi-log axes (figure $6 a$ ), an exponential growth $\left(\sim \mathrm{e}^{2 t / \tau}\right)$ is observed in the east for the smallest $D_{0}$ class with a time $\tau$ of $\sim 12$ days, from roughly a few days to three weeks. Whether a similar regime also occurs in the west for the smallest $D_{0}$ class is not clear (the corresponding error range is obviously underestimated because of correlated samples).

On the linear plots giving $\left(D^{2}\left(t, D_{0}\right)\right)^{1 / 3}$ as a function of time (figure $6 b$ ), it is possible to identify a quasi-linear growth up to 60 days in the west and 120 days (or perhaps more) in the east. Using equation (1) we obtain an estimate of $a=c \varepsilon^{1 / 3}$ as (3/2) $\times$ slope, whence the approximate values $a_{\mathrm{W}} \approx 1.4 \times 10^{-3} \mathrm{~m}^{2 / 3} \mathrm{~s}^{-1}\left(1.2 \times 10^{-3} \mathrm{~m}^{2 / 3} \mathrm{~s}^{-1}\right.$ with the original data set) for the west and $a_{\mathrm{E}} \approx 0.8 \times 10^{-3} \mathrm{~m}^{2 / 3} \mathrm{~s}^{-1}$ for the east, corresponding to slopes of $D^{2 / 3}$ versus time of $\sim 0.80 \mathrm{~km}^{2 / 3}$ day $^{-1}\left(\sim 0.70 \mathrm{~km}^{2 / 3} \mathrm{day}^{-1}\right.$ with the original data set) and $\sim 0.46 \mathrm{~km}^{2 / 3} \mathrm{day}^{-1}$ respectively. This supports a Richardson regime as given by (1).

To test how well such a law fits the experimental data and find out if there is an asymptotic $\left(\alpha t^{3}\right)$ regime, dispersion versus time is represented on a log-log plot. Figure 7 gives the dispersion behaviour for six initial separation classes chosen among the twelve $D_{0}$ classes (spanning a distance range from 0 to $120 \mathrm{~km}$, see table 1 ). Except for the first $D_{0}$ class, the Richardson law (1) fits the data qualitatively well, 
(a)

Western floats

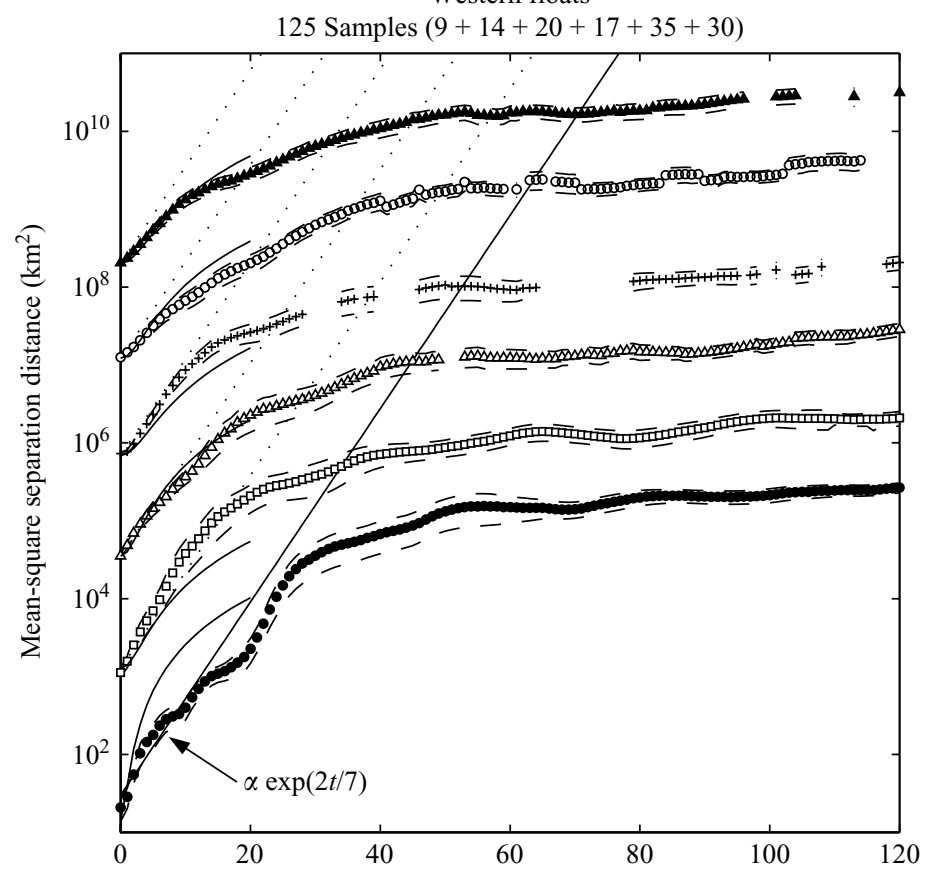

Eastern floats

83 Samples $(16+16+12+6+17+16)$

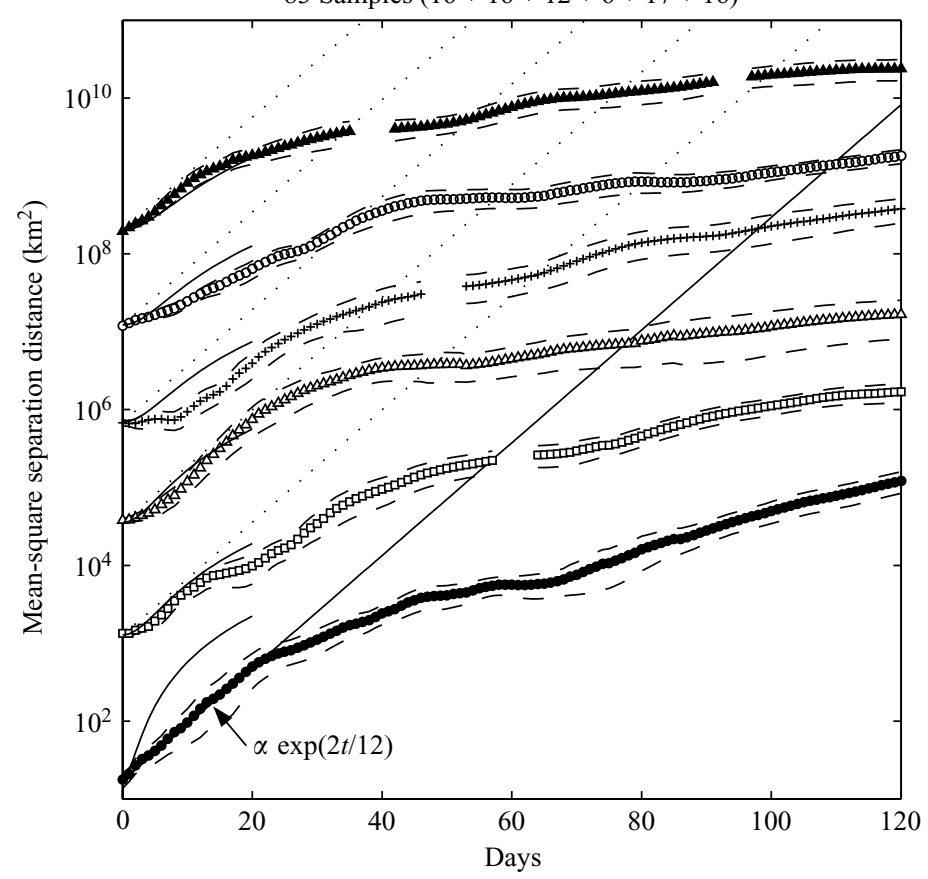

10 day re-initialization

Figure 6(a). For caption see facing page. 
(b)

Western floats

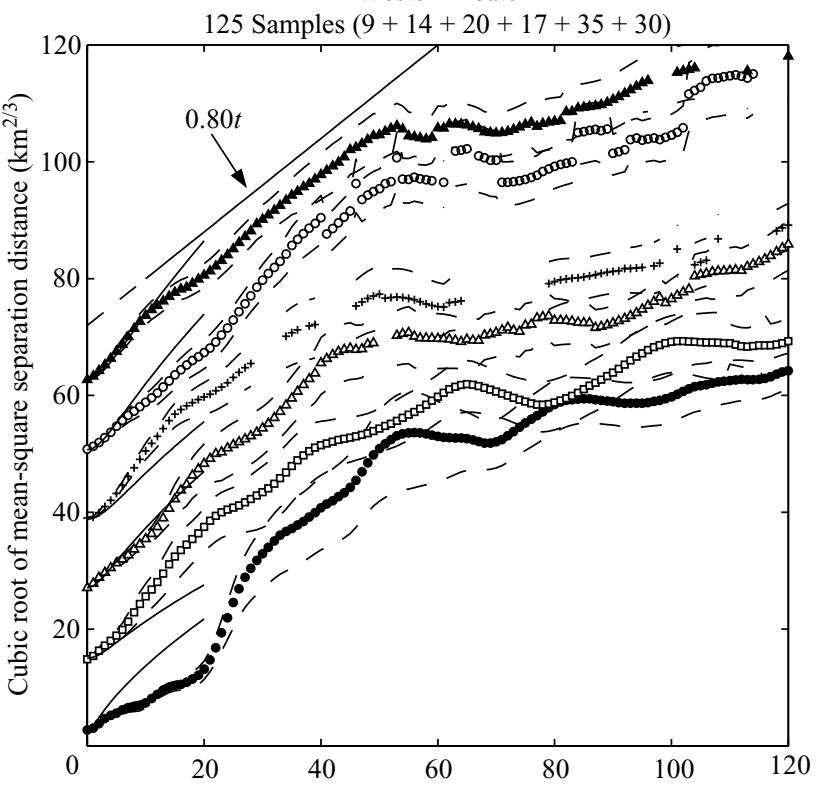

Eastern floats

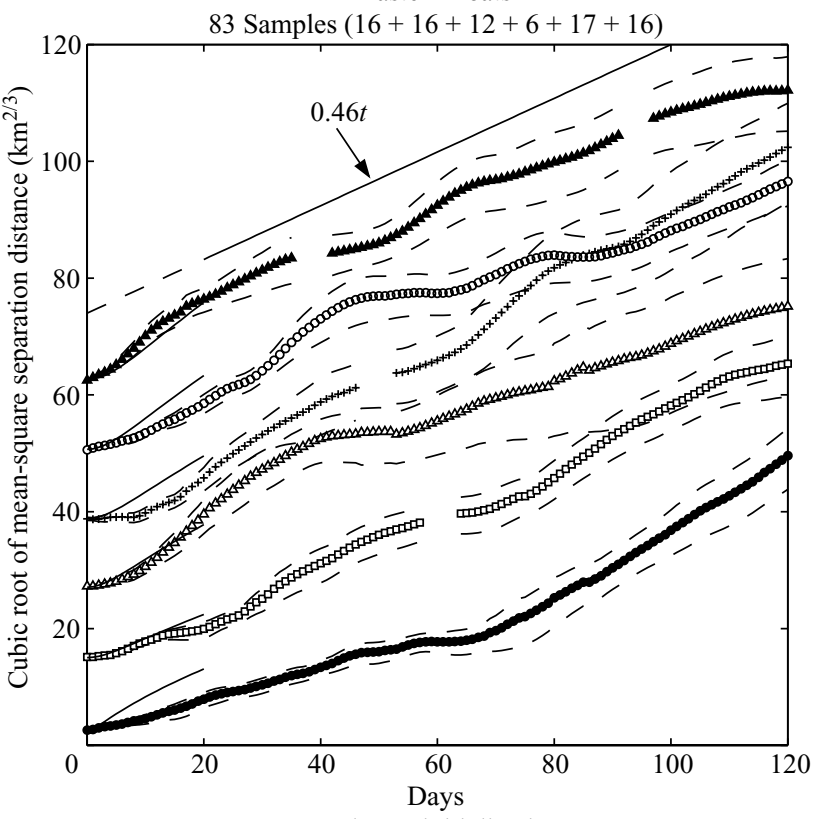

10 day re-initialization

FiguRE 6. (a) Mean-square separation distance $D^{2}\left(t, D_{0}\right) \equiv\left\langle D^{2}\left(t, \boldsymbol{D}_{0}\right)\right\rangle$ as a function of time and initial separation for the western (reduced) and eastern data sets. $D_{0}$ is within one of the six classes of distance [0, 7.5[,[7.5, 15[, [15, 22.5[,[22.5, 30[, [30, 40[ and [40, 50[km. Corresponding curves are given by solid dots, open squares, open triangles, crosses, open dots and solid triangles respectively. For readability $D^{2}$ values corresponding to different $D_{0}$ are shifted by a power of ten. Broken lines indicate \pm one standard deviation on the mean. The fit $D_{0}^{2}+\left\langle\left\|\delta \boldsymbol{V}_{0}\right\|^{2}\right\rangle t^{2}$ valid for small $t$ is plotted for $0 \leqslant t<20$ days. Only for the smallest class of initial distance and in the east does an exponential law stand out clearly. $(b) D^{2 / 3}\left(t, D_{0}\right)$ as a function of time and initial separation (same data and classes of distance as $(a)$ ). For readability $D^{2}$ values corresponding to different $D_{0}$ are shifted by a multiple of ten. 

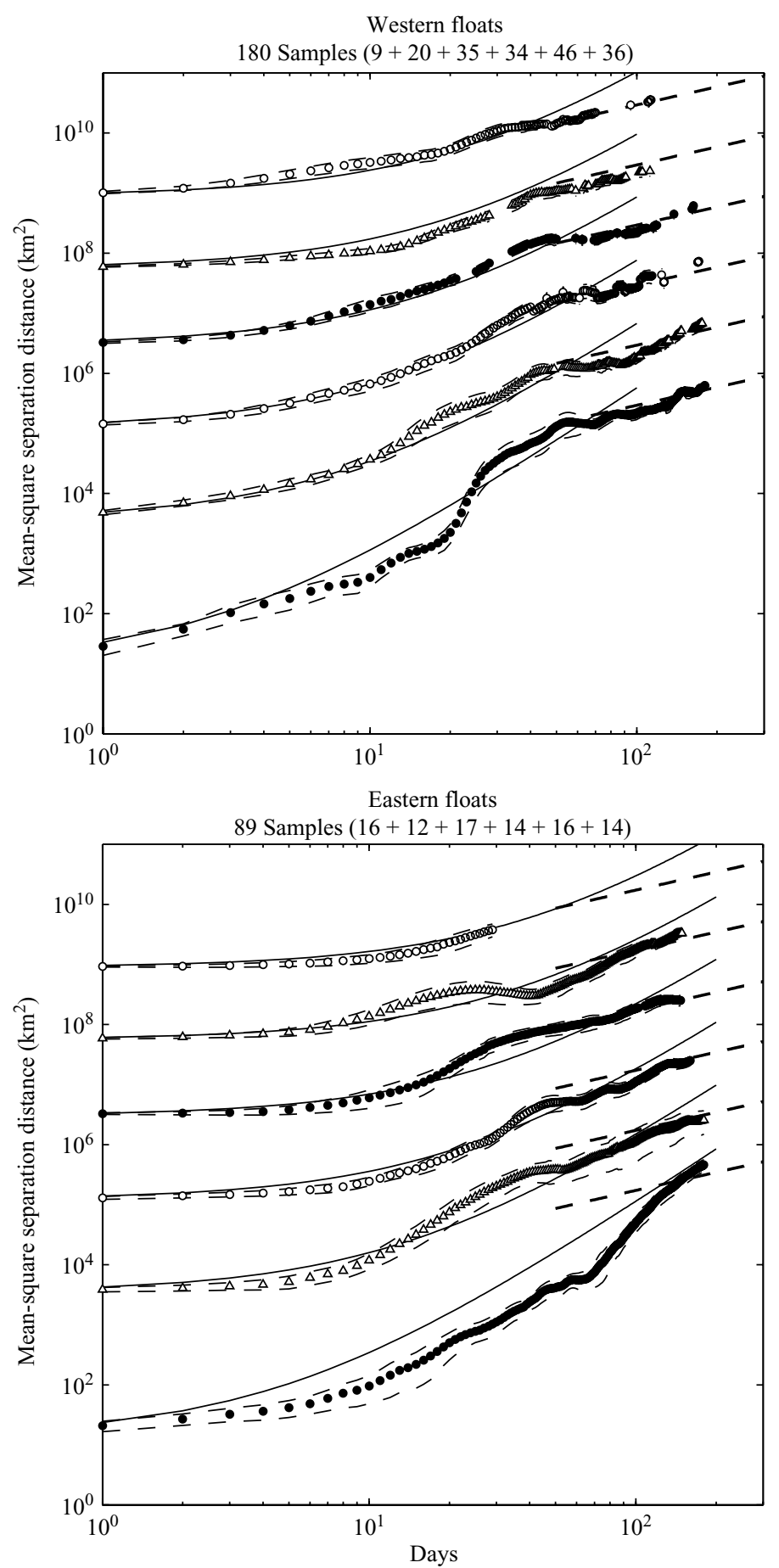

FIgURE 7. Mean-square separation distance $D^{2}\left(t, D_{0}\right)$ versus time and $D_{0}$, for the western (reduced) and eastern data sets. Six $D_{0}$ classes are shown (from bottom to top, $D_{0} \in[0,7.5[$, [15, 22.5[, [30, 40[, [50, 60[, [70, 80[ and [90, 100[ km). Richardson law $\left(D^{2}=\left(D_{0}^{2 / 3}+2 a t / 3\right)^{3}\right.$ with $a_{\mathrm{W}}=1.4 \times 10^{-3} \mathrm{~m}^{2 / 3} \mathrm{~s}^{-1}$ and $\left.a_{\mathrm{E}}=0.8 \times 10^{-3} \mathrm{~m}^{2 / 3} \mathrm{~s}^{-1}\right)$ and large time Taylor law $\left(D^{2}=4 K(\infty) t\right.$ with $K_{W}(\infty)=8.5 \times 10^{3} \mathrm{~m}^{2} \mathrm{~s}^{-1}$ and $K_{\mathrm{E}}(\infty)=5 \times 10^{3} \mathrm{~m}^{2} \mathrm{~s}^{-1}$ ) are also plotted. For readability $D^{2}$ values corresponding to different $D_{0}$ are shifted by a power of ten. Broken lines indicate \pm one standard deviation on the mean. 
before the random walk regime $(4 \mathrm{Kt}$ ) occurs ultimately. In agreement with equation (1), an asymptotic $t^{3}$ regime is only obtained after $D^{2}\left(t, D_{0}\right)$ becomes much greater than $D_{0}^{2}$.

Two-particle diffusivity $Y\left(t, D_{0}\right)$ is calculated by finite time differencing $D^{2}\left(t, D_{0}\right)$ and is plotted as a function of $D\left(t, D_{0}\right)$ on figure 8. Averaging individual values within distance intervals reveals, as expected, a reasonable fit to the law $Y(t)=d \chi^{1 / 3} D^{2}$ (with $\left(d \chi^{1 / 3}\right)^{-1}=\tau=12$ days in the east) and to the law $Y(t)=a D^{4 / 3}$ (with $a_{W}=1.4 \times$ $10^{-3} \mathrm{~m}^{2 / 3} \mathrm{~s}^{-1}$ and $\left.a_{\mathrm{E}}=0.8 \times 10^{-3} \mathrm{~m}^{2 / 3} \mathrm{~s}^{-1}\right)$. Although roughly confirmed in the east, the exponential (or $D^{2}$ ) regime is not resolved in the west, perhaps due to the paucity of data for small distances or because it simply does not exist. In the east, the transition to the Richardson (or $D^{4 / 3}$ regime) appears at separations between roughly 20 and $30 \mathrm{~km}$. With 30 day or even 60 day re-initializations, the two regimes (in the east) and the corresponding statistical laws, are similarly resolved (open squares or open triangles in figure 8). This gives support for our 10 day re-initialization procedure.

Using the original time series without re-initializations, we have also computed the doubling times $T\left(D_{\mathrm{n}}\right)$, defined as the mean time for a particle pair separation to grow from $D_{\mathrm{n}}$ to $2 D_{\mathrm{n}}$ (with $D_{1}=3 \mathrm{~km}$ and absorbing barriers). In the east, $T \sim 9$ days (or $\tau=T / \ln 2 \sim 13$ days) for the first four estimates, then varies roughly as $D^{2 / 3}$ for the next three estimates. In the west, $T$ varies very roughly as $D^{2 / 3}$ for the seven estimates, supporting the Richardson regime but not an exponential growth.

We had thought of obtaining an independent check of the statistical laws relating $Y$ to the distance by calculating a relative diffusivity $Y_{\mathrm{E}}(D)$ as the mean of $\boldsymbol{D}(t)$. $\delta \boldsymbol{V}\left(t, \boldsymbol{D}_{0}\right)$ from all pairs with the same $D$ (in practice of course within a small interval $\left[D, D+\mathrm{d} D\left[\right.\right.$ ), irrespective of time and initial separation $D_{0}$. By so doing we would obtain a large number of samples (without re-initializations). Being calculated with what amounts to an Eulerian operation, $Y_{\mathrm{E}}(D)$ is clearly different from our previous average, conditional on initial positions. In fact, when this unconditional averaging is carried out with our data, $Y_{\mathrm{E}}(D)$ is roughly half $Y(D)$. The following argument gives the origin of the difference. Considering the realizations $D\left(t, D_{0}\right)$ with $D_{0}$ fixed (in practice within a small interval $\left[D_{0}, D_{0}+\mathrm{d} D_{0}[)\right.$, one observes that $Y_{\mathrm{E}}\left(D, D_{0}\right)$ is generally smaller than $Y\left(D, D_{0}\right)$ for $D$ less than $100-200 \mathrm{~km}$. This is caused by some of the separation-distance time series that remained fluctuating in this distance range, whence a negligibly small contribution to $Y_{\mathrm{E}}$ because $\sum \mathrm{d} D^{2} / \mathrm{d} t=D_{\mathrm{k}+1}^{2}-D_{\mathrm{k}}^{2}$ (if $D^{2}$ increases) is cancelled by $\sum \mathrm{d} D^{2} / \mathrm{d} t=D_{\mathrm{k}}^{2}-D_{\mathrm{k}+1}^{2}$ (as $D^{2}$ decreases). Meanwhile, the other separation-distance time series grow much more rapidly, whence $\left\langle D^{2}\left(t, D_{0}\right)\right\rangle^{1 / 2}$, i.e. the r.m.s. average (at a given time) of both 'slow' and 'fast' separation distances, which swiftly leaves the range of $D$ less than $100-200 \mathrm{~km}$. This explains why $Y_{\mathrm{E}}(D)$, which is obtained after averaging of all the $D_{0}$ contributions, is smaller than $Y(D)$ for $D$ less than $100-200 \mathrm{~km}$, but (loosely) tends to $Y(D)$ for greater distance. It is worth noting, however, that the shape of $Y_{\mathrm{E}}$ versus $D$ is not very different from that obtained from the proper conditional averages. Whether $Y_{\mathrm{E}}$ is of physical significance and if so, how it relates to $Y$, remains to be studied.

Let us now suppose that for $D_{0}>30 \mathrm{~km}$, the Richardson law (1) applies from $t=0$ (possibly discarding the first few days, if necessary) until the time the Taylor or random walk regime is reached. One can say that the asymptotic $t^{3}$ regime is reached only for a short time period (e.g. a few days before the Taylor regime). But let us make a shift of the time, given by $\tilde{t}=t+t_{*}=t+3 D_{0}^{2 / 3} / 2 a$. Then (1) can be rewritten as $D^{2}(t)=8 a^{3} \tilde{t}^{3} / 27$ and now one can say that the regime is perfectly asymptotic (in $\tilde{t}^{3}$ ). So shifting in time the different dispersion curves (such as given in figure 7 ) 

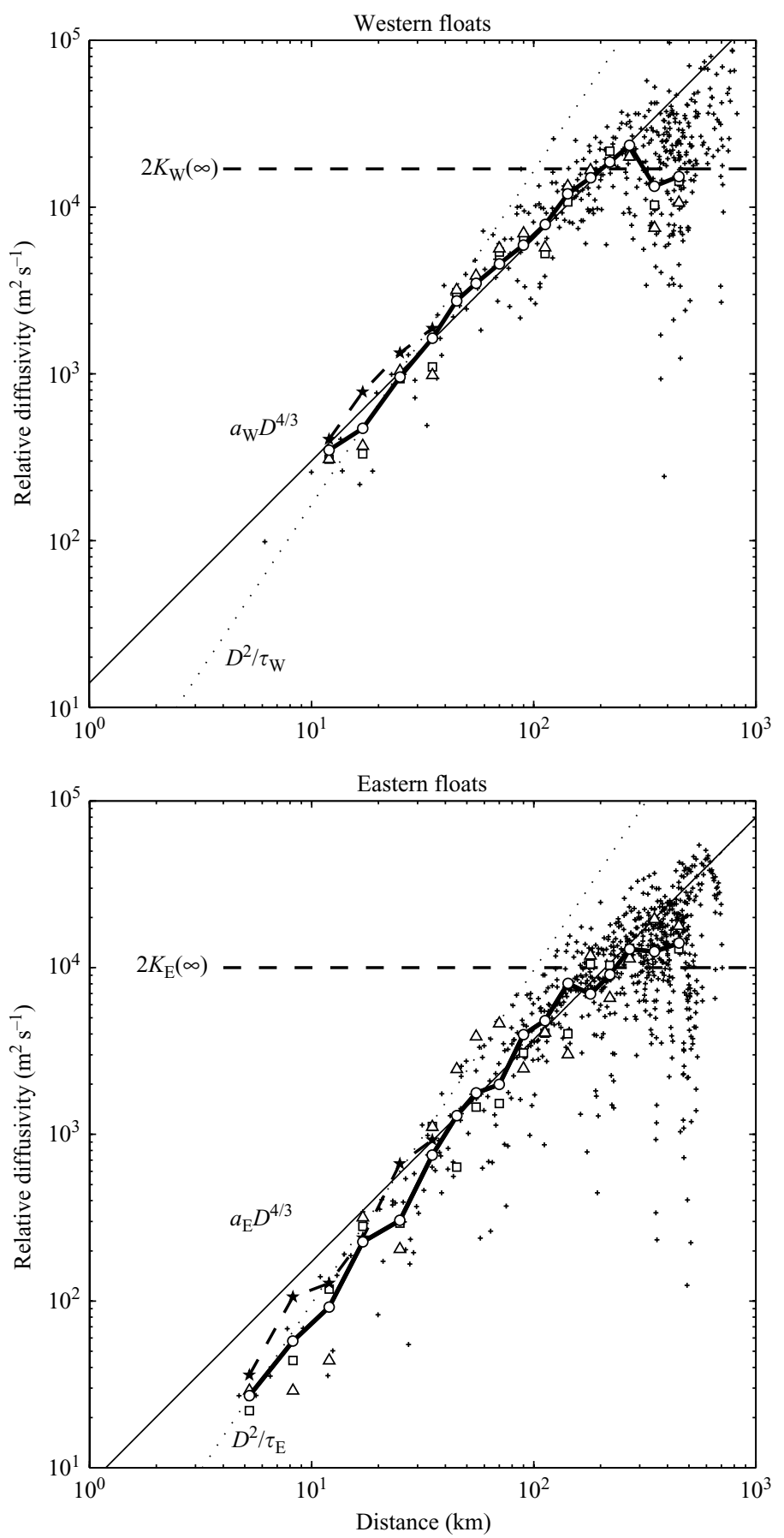

FIgURE 8. Relative diffusivity $Y\left(t, D_{0}\right)=\frac{1}{2}(\mathrm{~d} / \mathrm{d} t) D^{2}\left(t, D_{0}\right)$ versus $D$. Crosses give individual estimates obtained by finite differentiating the twelve mean-square separation distance time series corresponding to the twelve $D_{0}$ classes given in table 1 and for 10 day re-initialized time series. Open dots are weighted averages over values found within distance intervals. Open squares and triangles give the weighted averages for 30 day and 60 day re-initialized time series respectively. Stars give the corresponding evolution from the portions selected with the minimum separation criterion. The $a D^{4 / 3}$ and $D^{2} / \tau$ laws are given with $a_{\mathrm{W}}=1.4 \times 10^{-3} \mathrm{~m}^{2 / 3} \mathrm{~s}^{-1}$ and $a_{\mathrm{E}}=0.8 \times$ $10^{-3} \mathrm{~m}^{2 / 3} \mathrm{~s}^{-1}$, and with $\tau_{\mathrm{W}}=7$ days and $\tau_{\mathrm{E}}=12$ days respectively. 
corresponding to the eight $D_{0}$ classes with $30 \leqslant D_{0}<120 \mathrm{~km}$, and averaging, we obtain a unique curve (independent of $D_{0}$ ), given in figure 9. The different temporal shifts, calculated from $a_{\mathrm{W}}$ and $a_{\mathrm{E}}$ are given in the caption of figure 9. Since these composite plots are constructed under the assumption of a cubic regime, they do not prove its existence, but show that the data agree with such a regime. They combine the results obtained so far: if we assume a transition scale near $25 \mathrm{~km}$ (thus corresponding to a wavelength of about $150 \mathrm{~km}$ ), there is a possible exponential dispersion at smaller scales, a clear-cut Richardson regime over the $40-300 \mathrm{~km}$ distance range and a random walk regime reached for greater $D$, faster in the west (after 2 months) than in the east (after 4 months). The possibility that the western dispersion for the smallest $D_{0}$ class may follow a Richardson regime is not ruled out (solid dots or stars on figure 9), whereas the corresponding eastern dispersion departs clearly from the $\tilde{t}^{3}$ curve, over the first ten days (either with 10 day or 60 day re-initializations, or even with portions selected with the minimum criterion).

The mean-square relative velocities $\left\langle\left\|\delta \boldsymbol{V}\left(t, \boldsymbol{D}_{0}\right)\right\|^{2}\right\rangle$ are given on figure 10 for the twelve $D_{0}$ classes. It is pleasing to note that they show the same kind of time evolution as those from Babiano et al. (1990, their figure 2a). Although it has been argued that within the inverse energy cascade range, $\left\langle\left\|\delta \boldsymbol{V}\left(t, \boldsymbol{D}_{0}\right)\right\|^{2}\right\rangle$ should vary as $t$ (Monin \& Yaglom 1975; Babiano et al. 1990), this is not visible here, nor in Babiano et al.'s (1990) or Kowalsky \& Peskin's (1981) numerical results, probably because the assumptions underlying the theoretical result are too stringent. Nevertheless, when $t$ is large, the mean-square relative velocity equilibrates to four times the kinetic energy in agreement with (3), giving rough estimated values of 30 (60) days for the pair relative velocity decorrelation time scale in the west (east). Since as $t \rightarrow 0$ the mean-square relative velocity tends towards $Z D_{0}^{2}$, we can estimate the enstrophy $Z$, obtaining values between 0.6 and $0.06 \mathrm{day}^{-2}$ in the west, and between 0.2 and 0.02 $\mathrm{day}^{-2}$ in the east. A better estimate for $Z$ should come from the characteristic time of relative dispersion $\tau_{\mathrm{y}}\left(t, D_{0}\right)=D^{2}\left(t, D_{0}\right) / Y\left(t, D_{0}\right)$ as a function of $D$. Babiano et al. (1990) have shown that it reaches a minimum equal to $2 / Z^{1 / 2}$ over the distance range of the exponential regime. Figure 11 shows the results for $\tau_{y}$, in approximate agreement with such an interpretation, providing $Z \approx 0.16 \mathrm{day}^{-2}$ in the west and $0.04 \mathrm{day}^{-2}$ in the east (or a minimum characteristic time $\tau$ of 5 days in the west and 10 days in the east).

The separation probability density functions (p.d.f.) were estimated at 10 day intervals. Figure 12 gives the p.d.f.s (rescaled so that their variance is unity) for initial separations in the ranges $0 \leqslant D_{0}<7.5 \mathrm{~km}$ and $30 \leqslant D_{0}<40 \mathrm{~km}$, for the east (western p.d.f.s, not shown, are similar but slightly noisier). The comparison with an assumed Gaussian distribution for $\boldsymbol{D}$, i.e. a Rayleigh distribution for $\|\boldsymbol{D}\|(\boldsymbol{D}$ is a sample separation, not a r.m.s. value), is encouraging for large $D_{0}$, but not at all for small $D_{0}$, whose observed distribution contains many more small separations. This result agrees with Davis (1985), who instead used surface drifters off the California coast. Highly kurtosed distributions were also obtained experimentally (with $10^{4}$ pairs) by Jullien et al. (1999), for small $D_{0}\left(=D_{I} / 50\right)$. Their distributions corresponding to different times for which the Richardson scaling holds, i.e. for $D\left(t, D_{0}\right)$ greater than (or of the order of) $D_{I}$, reveal that the separation process is self-similar in time in the range considered (this seems likely for our data too). A best fit for the tails of their p.d.f.s (also rescaled), is $\exp \left(-\alpha s^{1 / 2}\right)$ with $\alpha \sim 2.6$, thus strongly non-Gaussian. As a comparison the distribution given by $2 \exp \left\{-2 s^{1 / 2}\right\}$ plotted on figure 12 (small $D_{0}$ case), is seen to fit roughly our data. Due to our reduced number of samples however, we cannot be more quantitative. 

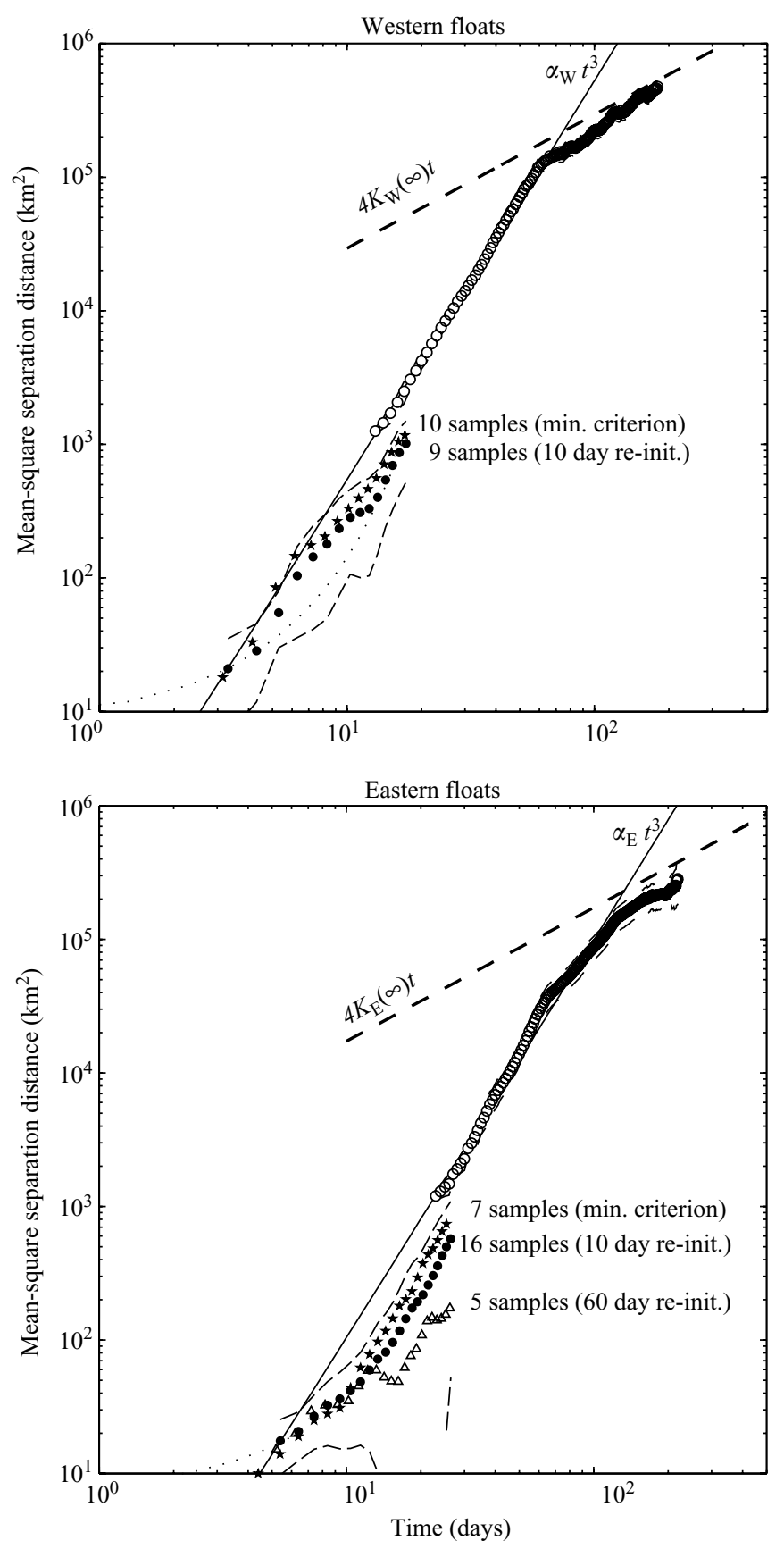

FIGURE 9. Mean-square separation distance versus time obtained by averaging at a given time, the individual mean-square separation distance curves corresponding to $D_{0}>30 \mathrm{~km}$, but shifted in time as explained in the text, so that they start as if they were already in the asymptotic Richardson regime. Actually the shifts are 13, 16, 18, 20, 22, 24, 26 and 29 days in the west and $23,27,31,35,39,42,45$ and 50 in the east. Broken lines indicate \pm two standard deviations on the mean. Solid dots (open triangles) give the mean-square separation distance, for the smallest $D_{0}$ class for the 10 day (60 day) reinitialized time series, and shifted in time as if it had started in the asymptotic Richardson regime. Stars give the corresponding evolution for the portions selected with the minimum separation criterion. Clearly the eastern dispersion does not follow a $\tilde{t}^{3}$ law over the first 10 days. 


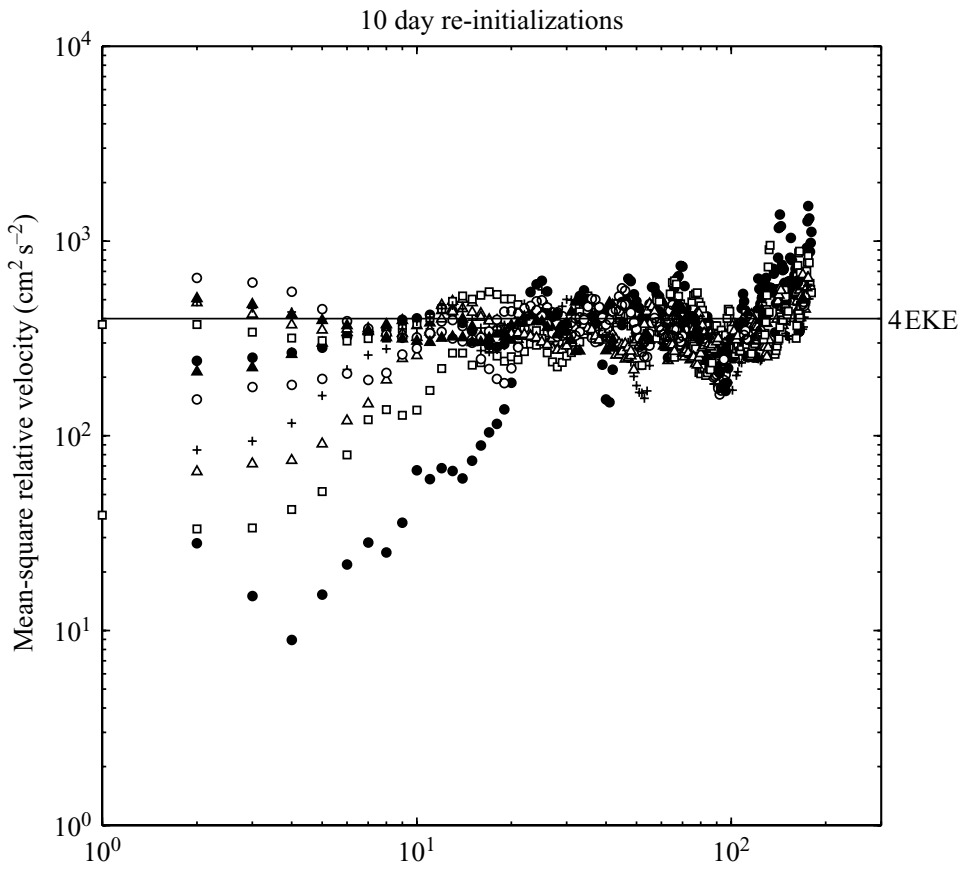

Eastern floats

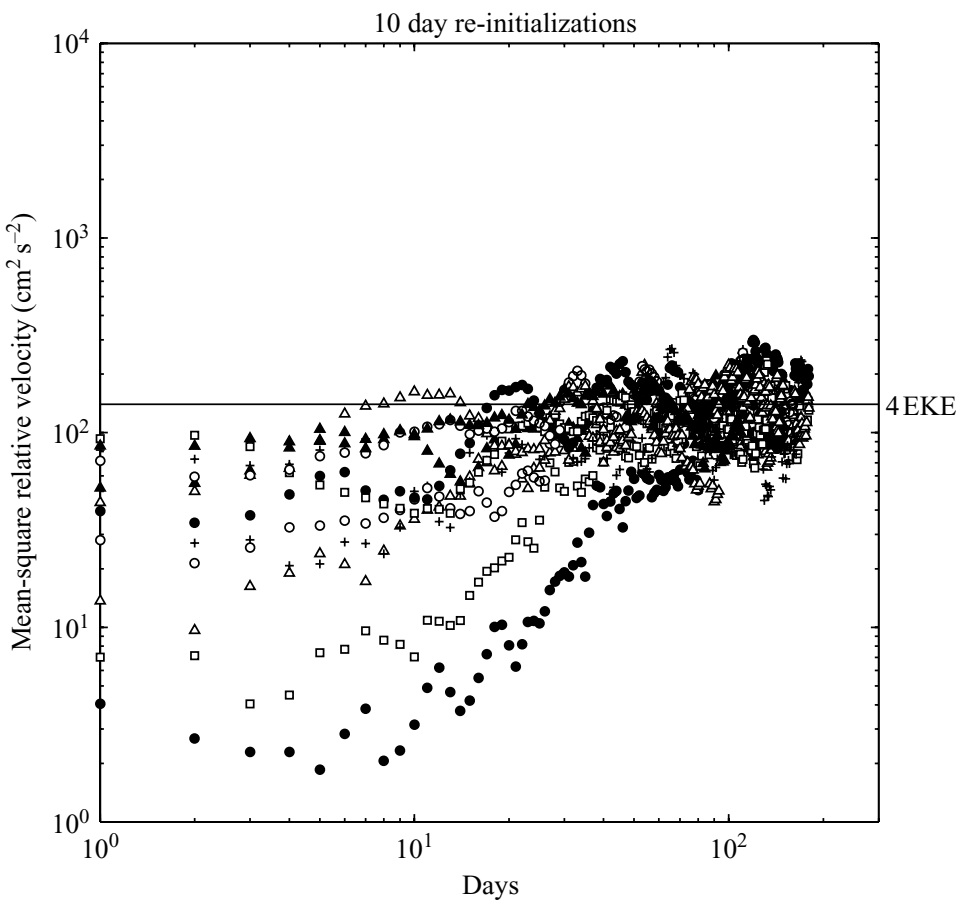

Figure 10. Mean-square relative velocity $\left\langle\left\|\delta \boldsymbol{V}\left(t, \boldsymbol{D}_{0}\right)\right\|^{2}\right\rangle$ as a function of time. Symbols corresponding to the first six $D_{0}$ classes are as in figure 6 . The same symbols are used similarly for the last six $D_{0}$ classes (table 1). 
Western floats

10 day re-initializations

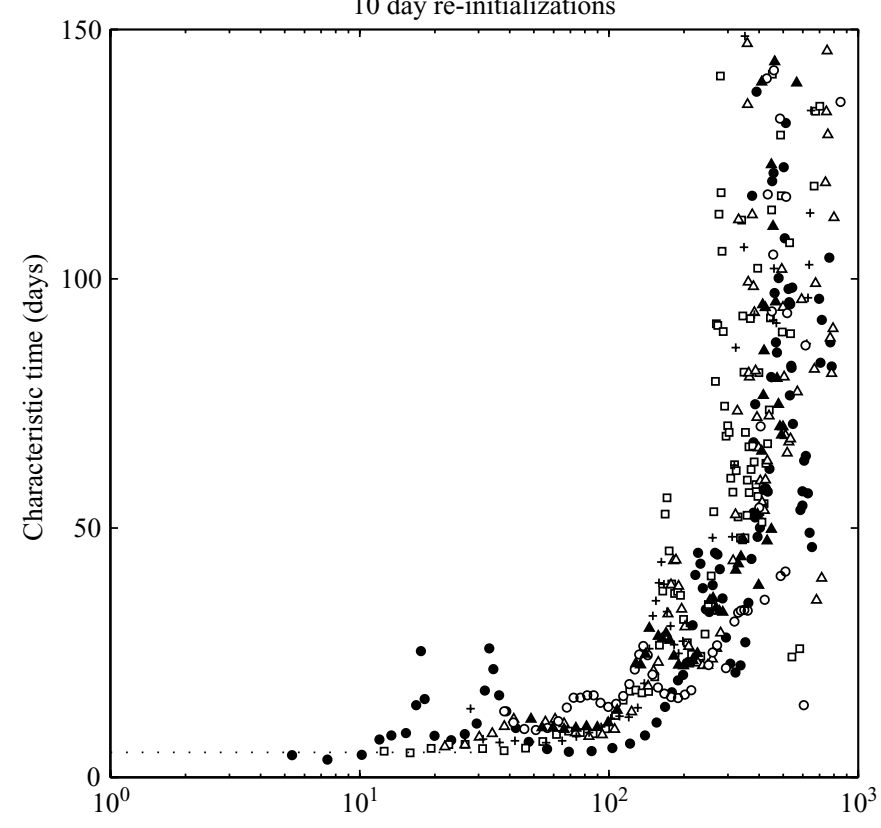

Eastern floats

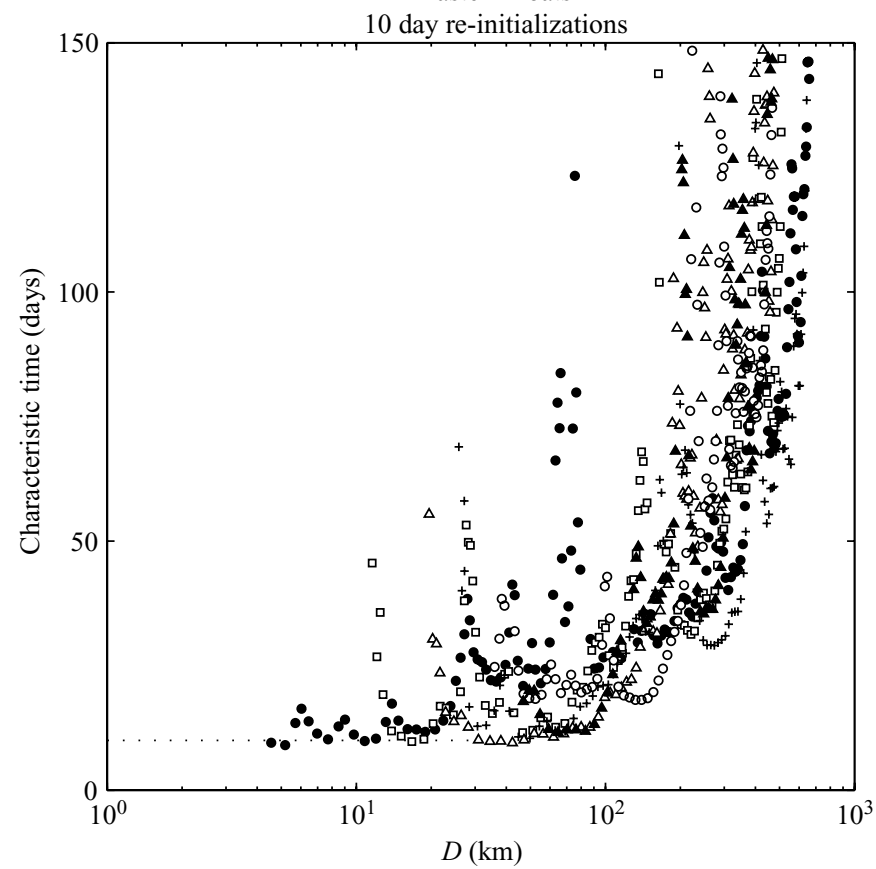

FIgURE 11. Characteristic time $\tau_{y}\left(t, D_{0}\right)=D^{2}\left(t, D_{0}\right) / Y\left(t, D_{0}\right)$ as a function of $D$ and $D_{0}$ and for the first six $D_{0}$ classes $\left(0 \leqslant D_{0}<50 \mathrm{~km}\right)$.

\section{Discussion}

At scales less than the Rossby radius of deformation (here for $D<25 \mathrm{~km}$ ), our observation of a possible exponential regime gives support for the traditional enstrophy 
Eastern floats
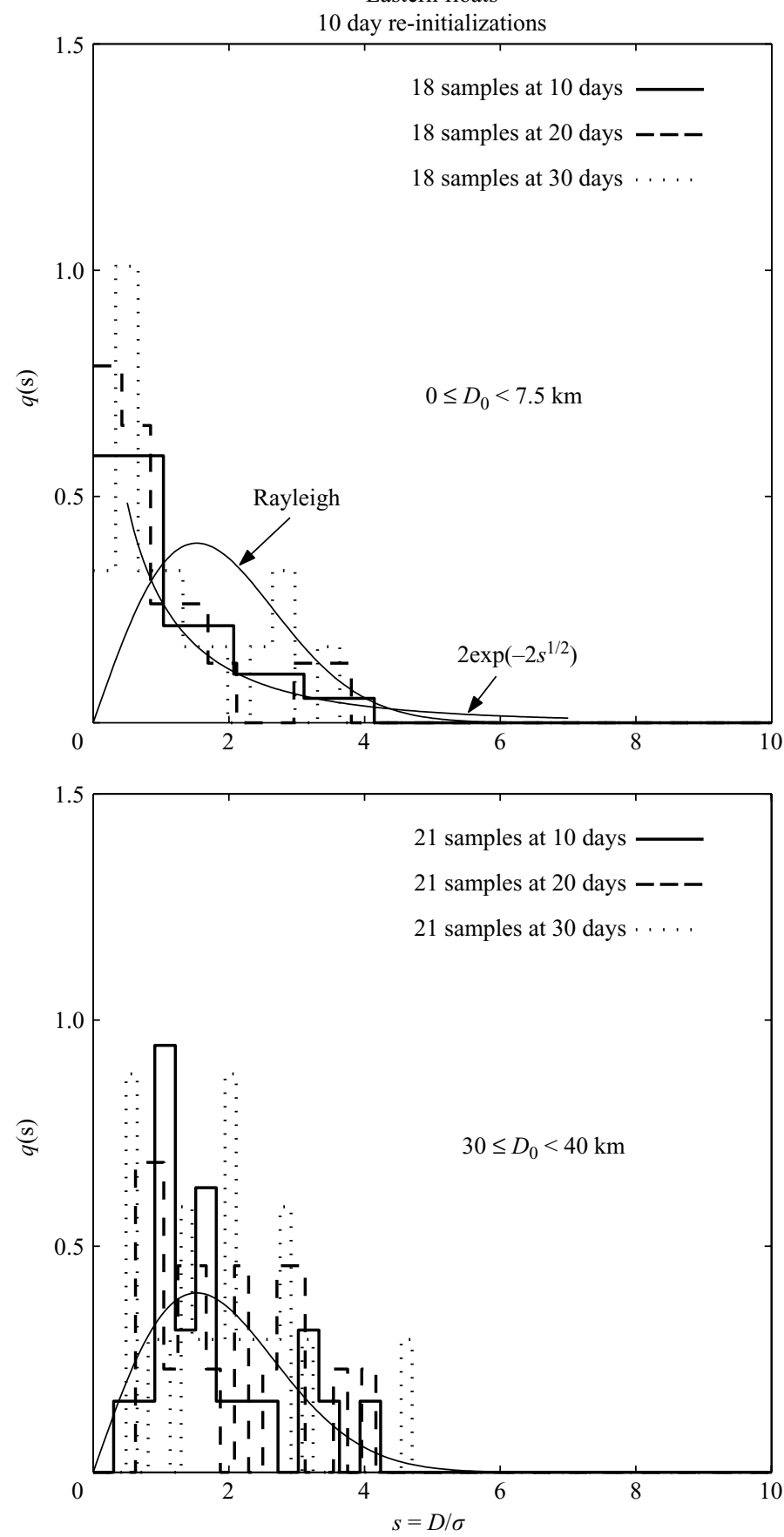

FiguRE 12. Probability density functions $q(s, t)$ of rescaled pair separations $s=D / \sigma$ (here $D=\|\boldsymbol{D}\|)$ after 10,20 and 30 days, for small $\left(0 \leqslant D_{0}<7.5 \mathrm{~km}\right)$ and large $\left(30 \leqslant D_{0}<40 \mathrm{~km}\right)$ initial separations, and for the eastern floats. $p(D, t)$ is the original p.d.f., whence $q(s, t)=$ $\sigma p(\sigma s, t)$ where $\sigma^{2}$ is the variance of $D$. The Rayleigh distribution (rescaled) is given by $\lambda s \exp \left(-\lambda s^{2} / 2\right)$ with $\lambda=(4-\pi) / 2$. 
cascade to small scales. However in geostrophic turbulence long-lived coherent vortices are found, along with more random fluctuations (McWilliams 1984). These coherent features are the signature of elliptic regions. Since numerical simulations of twodimensional turbulence by Elhmaidi, Provenzale \& Babiano (1993) indicate that hyperbolic regions occupy a larger domain than elliptic ones, they will be favoured by an initial seeding of particles over a regular grid. Thus, the exponential separation may simply result, for small enough time and separation, from the pre-eminence of these hyperbolic regions.

The recent analysis of Lacasce \& Ohlmann (2003) of surface trajectories in the Gulf of Mexico clearly shows an exponential growth with $D_{0} \leqslant 1 \mathrm{~km}$ and for separations smaller than $40 \mathrm{~km}$. Although such drifters may experience Ekman drift, windage and surface flow convergence, these authors argue that an enstrophy regime is possible for $D$ less than $R_{\text {int }}$ ( $45 \mathrm{~km}$ in the Gulf of Mexico). The compilation of subsurface float experiments by Lacasce \& Bower (2000) did not indicate an exponential regime and their first explanation (supported by the above surface results) was that not enough pairs with small initial separation were observed. This may be why no exponential growth was revealed in our western cluster. A large number of pairs is needed to ensure the results are statistically significant: our nine pairs in the west were not enough. Only with a mean $D_{0} \sim 4 \mathrm{~km}$ and 16 pairs in the east were we able to obtain an exponential growth. Another recent analysis by Colas (2003) of subsurface float pair separations (with $D_{0} \leqslant 10 \mathrm{~km}$ ) in the eastern North Atlantic also supports the idea of an exponential regime but only marginally. The difference between our two clusters may also be of topographic origin: there are examples of pairs which separate when hitting seamounts in the east, but none in the west. The exponential regime found for our eastern cluster in the ocean interior needs to be confirmed with better statistics, and far from topographic influences.

Closer to a realistic oceanic case than Babiano et al.'s (1990) results, numerical simulations of pair dispersion in an eddy-resolving wind-driven, stratified oceanic model carried out by Berloff et al. (2001) confirms the existence of an initial exponential regime with values of $\tau$ of order 10 days in regions with eddy kinetic energy of order $100 \mathrm{~cm}^{2} \mathrm{~s}^{-2}$. This agrees with our experiment since $\tau$ is 12 days for eastern pairs. Lagrangian integral time scales $T_{L}$ have approximately the same values (7-10 days in the east, 5-6 days in the west) whereas the western cluster has eddy kinetic energy (EKE) three times larger than the eastern cluster $\left(100 \mathrm{~cm}^{2} \mathrm{~s}^{-2}\right.$ versus $35 \mathrm{~cm}^{2} \mathrm{~s}^{-2}$ ). If we apply the parameterization given in OCV (that seems to fit mid-latitude oceanic data), that is $K_{11}+K_{22}=K(\infty) \approx \gamma R_{i n t} \operatorname{EKE}^{1 / 2}$ (with $\gamma \sim 3$ at $700-1000 \mathrm{~m}$ depth) and if we assume that $\tau \approx T_{L}$, we obtain a prediction of the exponential law for any region: since $K(\infty)=2 E K E T_{L}$ we have $2 \tau \approx \gamma R_{\text {int }} \mathrm{EKE}^{-1 / 2}$. For the EOLE and TWERL atmospheric experiments, Morel \& Larcheveque (1974) and Er-El \& Peskin (1981) respectively give EKE $\approx 150 \mathrm{~m}^{2} \mathrm{~s}^{-2}, R_{\text {int }} \approx O(1000) \mathrm{km}$ (at mid-latitudes). Their observed value of $\tau \approx 3$ days is of the order of the predicted value $\tau \sim 1.5$ days.

Since the Richardson regime must be found in two-dimensional turbulence for distances larger than those associated with the exponential regime, coarse resolution data are sufficient to detect it. Lacasce \& Bower (2000) confirmed the $D^{4 / 3}$ law but only for experiments in the western basin of the North Atlantic. The impressive older data compilation by Okubo (1971) combines two- and three-dimensional aspects making comparisons difficult. For large-scale atmospheric data, the Richardson regime was not examined by Morel \& Larcheveque (1974), while much scatter remains in Er-El \& Peskin's (1981) $t^{3}$ fit to their data. This may be due to the factor of 10 
which exists between the scales of atmospheric and oceanic motions making the homogeneous and stationary turbulence assumptions more difficult to justify for the larger-scale atmospheric motions. The results of two-dimensional numerical forced experiments comply more closely with expectations. The $t^{3}$ asymptotic regime was observed by Babiano et al. (1990) but only for $D_{0}$ much smaller than their injection scale. Furthermore the regime is reached later as $D_{0}$ increases (their figure 4). We point out that this behaviour may be explained simply by the form $\left(D_{0}^{2 / 3}+2 a t / 3\right)^{3}$ of the integrated Richardson law (1). When $D_{0}$ is larger than the injection scale (the Rossby radius of deformation in our experimental context), the temporal law (1) is recovered over the whole time range and we propose therefore that this form (1) has to be preferred over the asymptotic $t^{3}$ limit when tests of the Richardson regime are sought. No such ambiguity appeared in Jullien et al.'s (1999) laboratory experiment: since their $D_{0}$ values were much smaller than their injection scale $D_{I}$, the $t^{3}$ asymptotic was reached as soon as $D$ was larger than $D_{I}$.

As relative dispersion in a mean shear may also produce a $t^{3}$ regime (e.g. Bennett 1987), Lacasce \& Bower (2000) suggested that some of their $D^{4 / 3}$ results could be caused by such an effect due to the proximity of strong mean currents. Given the isotropic character of the dispersion process in the TOPOGULF data (figure 5), this explanation, however, seems unlikely in our case.

The $D^{4 / 3}$ scaling for the diffusivity that we observe may well be associated with the existence of an inertial regime over the relevant scales $(40-300 \mathrm{~km})$. We have estimated values for $a=c \varepsilon^{1 / 3}$ to be about $0.8 \times 10^{-3} \mathrm{~m}^{2 / 3} \mathrm{~s}^{-1}$ and $1.4 \times 10^{-3} \mathrm{~m}^{2 / 3} \mathrm{~s}^{-1}$ respectively for the eastern and western cluster. Available estimates (Monin \& Yaglom 1975) vary between $10^{-4}$ and $10^{-2} \mathrm{~m}^{2 / 3} \mathrm{~s}^{-1}$, thus consistent with our estimates.

The quantity $\varepsilon$ in two-dimensional turbulence represents the rate at which kinetic energy is transferred up scale. Alves \& Colin de Verdière's (1999) numerical integrations of the nonlinear eddy generation process for the region analysed herein (the area of the Azores subtropical front) indicate that eddies appear initially through the baroclinic instability of the quasi-geostrophically balanced jet at wavelengths (scales) larger than $50(8) \mathrm{km}$ with a broad maximum at $150(25) \mathrm{km}$ (scale is more appropriate to compare with separation distance). After about 100 days, there is evidence of an upscale (inverse) energy cascade, the energy-containing wavelengths (scales) reaching $240(40) \mathrm{km}$. From this, we could conclude that the injection of eddy energy occurs towards the small-scale end of our postulated inertial range and that we witness evidence of an inverse energy cascade. The eddy equilibration does not reach the full barotropic state, however, a process which has been shown recently by Smith \& Vallis $(2001,2002)$ to be caused by the surface-intensified structure of the oceanic stratification which allows a significant part of baroclinic energy to remain near the scale $R_{\text {int }}$. With their interpretation in mind, the inertial eddy-eddy interactions that we infer in the main thermocline at scales larger than $R_{\text {int }}$ would be the result of an upscale energy cascade for the barotropic mode and a downscale cascade for the baroclinic modes. In order to compare with other experiments, we choose $\varepsilon$ to be the eddy kinetic energy conversion from the mean potential energy. Alves \& Colin de Verdière (1999) estimated this energy flux $\left(-g \overline{w^{\prime} \rho^{\prime}} / \rho_{0}\right)$ to be $10^{-10} \mathrm{~m}^{2} \mathrm{~s}^{-3}$ and when such a value is used as a proxy for $\varepsilon$, the constant $c$ of proportionality between $Y$ and $\varepsilon^{1 / 3} D^{4 / 3}$ is about 2 or 3 . Dedicated experiments on geostrophic turbulence and theory will be needed to test the value of this definition.

Finally, according to our data, the Gaussian distribution, proposed by Batchelor (1952), seems to be ruled out when $D_{0}$ is small (compared to the energy injection scale $\left.D_{I}\right)$ and for times when the Richardson regime begins to hold, that is when $D$ is order 
of or slightly greater than $D_{I}$ (30 days after launch in our experimental results), in agreement with the two-dimensional turbulence experimental results of Jullien et al. (1999) or the numerical simulations by Boffetta \& Sokolov (2002). However, the latter authors show that the distribution evolves to a Gaussian as time increases, but our data did not allow us to explore this. Whether the observed (and rescaled) oceanic distribution is identical to the one found in Jullien et al's experiment for $D_{0} \ll D_{I}$, and whether the Gaussian distribution is adequate for separations conditional upon an initial $D_{0} \geqslant D_{I}$, or at large time, must await confirmation until a larger observational data base is gathered.

We wish to thank Armando Babiano, Xavier Carton, Bach-Lien Hua and Jim McWilliams for discussions regarding this work, and all reviewers for helping us to clarify the paper.

\section{REFERENCES}

Alves, M. \& Colin de Verdière, A. 1999 Instability dynamics of a subtropical jet and applications to the Azores Front current systems: eddy driven mean flows. J. Phys. Oceanogr. 29, 837-864.

Arhan, M., Colin de Verdière, A. \& Mercier, H. 1989 Direct observations of the mean circulation at $48^{\circ} \mathrm{N}$ in the Atlantic Ocean. J. Phys. Oceanogr. 19, 161-181.

Babiano, A., Basdevant, C., Le Roy, P. \& SAdourny, R. 1990 Relative dispersion in two-dimensional turbulence. J. Fluid Mech. 214, 535-557.

Babiano, A., Basdevant, C. \& Sadourny, R. 1985 Structure functions and dispersion laws in two-dimensional turbulence. J. Atmos. Sci. 42, 942-949.

BAtchelor, G. K. 1952 Diffusion in a field of homogeneous turbulence, II. The relative motion of particles. Proc. Camb. Phil. Soc. 48, 345-362.

BatcheloR, G. K. 1953 The Theory of Homogeneous Turbulence. Cambridge University Press.

Bennett, A. F. 1984 Relative dispersion: local and nonlocal dynamics. J. Atmos. Sci. 41, 1881-1886.

Bennett, A. F. 1987 A Lagrangian analysis of turbulent diffusion. Rev. Geophys. 25, 799-822.

Berloff, P. S., McWilliams, J. C. \& Bracco, A. 2001 Material transport in oceanic gyres. Part 1: phenomenology. J. Phys. Oceanogr. 32, 764-796.

Boffetta, G. \& SoKolov, I. M. 2002 Statistics of two-particle dispersion in two-dimensional turbulence. Phys. Fluids 14, 3224-3232.

Charney, J. G. 1971 Geostrophic turbulence. J. Atmos. Sci. 28, 1087-1095.

Colas, F. 2003 Circulation et dispersion Lagrangiennes en Atlantique Nord-Est. Thèse de 3ème cycle de l'Université de Bretagne Occidentale.

Colin de Verdière, A., Mercier, H. \& Arhan, M. 1989 Mesoscale variability transition from the western to the eastern Atlantic along $48^{\circ}$ N. J. Phys. Oceanogr. 19, 1149-1170.

Corrsin, S. 1962 Theories of turbulent dispersion. In Mécanique de la Turbulence, pp. 27-52. CNRS, Paris.

DAVIS, R. E. 1985 Drifter observations of coastal surface currents during CODE: the statistical and dynamical views. J. Geophys. Rev. 90, C3, 4756-4772.

Elhmaidi, D., Provenzale, A. \& Babiano, A. 1993 Elementary topology of two-dimensional turbulence from a Lagrangian viewpoint and single-particle dispersion. J. Fluid Mech. 257, 533-558.

ER-El, J. \& Peskin, R. L. 1981 Relative diffusion of constant-level balloons in the Southern hemisphere. J. Atmos. Sci. 38, 2264-2274.

Gill, A. E. 1982 Atmosphere-Ocean Dynamics. Academic Press.

Jullien, M.-C., Paret, J. \& Tabeling, P. 1999 Richardson Pair dispersion in two dimensional turbulence. Phys. Rev. Lett. 82, 2872-2875.

Kowalsky, A. D. \& Peskin, R. L. 1981 Numerical simulation of relative dispersion in twodimensional, homogeneous, decaying turbulence. J. Fluid Mech. 109, 45-61.

Kraichnan, R. H. 1966 Dispersion of particle pairs in homogeneous turbulence. Phys. Fluids 9, 1937-1943.

Kraichnan, R. H. 1967 Inertial ranges in two-dimensional turbulence. Phys. Fluids 10, 1417-1423. 
Kraichnan, R. H. \& Montgomery, D. 1980 Two-dimensional turbulence. Rep. Prog. Phys. 43, 547-619.

Lacasce, J. H. \& Bower, A. 2000 Relative dispersion in the subsurface North Atlantic. J. Mar. Res. 58, 863-894.

Lacasce, J. H. \& Ohlmann J. C. 2003 Relative dispersion at the surface of the Gulf of Mexico. J. Mar. Res. 61, 285-312.

LARCHEVEQUe, M. 1983 Influence de la turbulence bidimensionnelle sur la diffusion. J. Méc. Théor. Appl., Special Issue, 271-292.

LARCHEVEQUe, M. \& Lesieur, M. 1981 The application of eddy-damped Markovian closures to the problem of dispersion of particle pairs. J. Méc. 20, 113-134.

LesIeUR, M. 1997 Turbulence in Fluids, 3rd edition. Kluwer.

LiN, J. T. 1972 Relative dispersion in the enstrophy-cascading inertial range of homogeneous two-dimensional turbulence. J. Atmos. Sci. 29, 394-396.

McWilliams, J. C., Brown, E. D., Bryden, H. L. et al. 1983 The local dynamics of eddies in the western North Atlantic. In Eddies in Marine Science, pp. 93-113. Springer.

McWilliams, J. C. 1984 The emergence of isolated coherent vortices in turbulent flow. J. Fluid Mech. 146, 21-43.

Monin, A. S. \& Yaglom, A. M. 1975 Statistical Fluid Mechanics. Vol. 2. The MIT Press.

Morel, P. \& Larcheveque, M. 1974 Relative dispersion of constant-level balloons in the $200 \mathrm{mb}$ general circulation. J. Atmos. Sci. 31, 2189-2196.

OвuкHOv, A. M. 1941 Energy distribution in the spectrum of turbulent flow. Izv. Akad. Nauk. SSSR, Ser. Geogr. Geofiz. 5, 453-466.

Okubo, A. 1971 Oceanic diffusion diagrams. Deep-Sea Res. 18, 789-802.

Ollitrault, M. 1994 The TOPOGULF experiment Lagrangian data. Repères Océan. $\mathrm{N}^{\circ} 7$ IFREMER.

Ollitrault, M. \& Colin de VerdiÈre, A. 2002a SOFAR Floats reveal mid-latitude intermediate North Atlantic general circulation Part I: a Lagrangian descriptive view. J. Phys. Oceanogr. 32, 2020-2033.

Ollitrault, M. \& Colin DE VerdiÈre, A. 2002 $b$ SOFAR Floats reveal mid-latitude intermediate North Atlantic general circulation Part II: an Eulerian statistical view. J. Phys. Oceanogr. 32, 2034-2053.

Richardson, L. F. 1926 Atmospheric diffusion on a distance-neighbour graph. Proc. R. Soc. Lond. A 110, 709-737.

Rhines, P. B. 1979 Geostrophic turbulence. Annu. Rev. Fluid Mech. 11, 401-441.

SAlmon, R. 1998 Lectures on Geophysical Fluid Dynamics. Oxford University Press.

SaWford, B. 2001 Turbulent relative dispersion. Annu. Rev. Fluid Mech. 33, 289-317.

Smith, K. S. \& Vallis, G. K. 2001 The scales and equilibration of midocean eddies: freely evolving flow. J. Phys. Oceanogr. 31, 554-571.

Smith, K. S. \& Vallis, G. K. 2002 The scales and equilibration of midocean eddies: forced dissipative flow. J. Phys. Oceanogr. 32, 1699-1720.

Stammer, D. 1997 Global characteristics of ocean variability estimated from regional Topex/Poseidon altimeter measurements. J. Phys. Oceanogr. 27, 1743-1769.

TaYlor, G. I. 1921 Diffusion by continuous movements. Proc. Lond. Math. Soc. 20, 196-212.

TrÉGUIER, A. M. \& HUA, B. L. 1988 Influence of bottom topography on stratified quasi-geostrophic turbulence in the ocean. Geophys. Astrophys. Fluid Dyn. 43, 265-305.

WunsCH, C. 1997 The vertical partition of oceanic horizontal kinetic energy. J. Phys. Oceanogr. 27, $1770-1794$. 